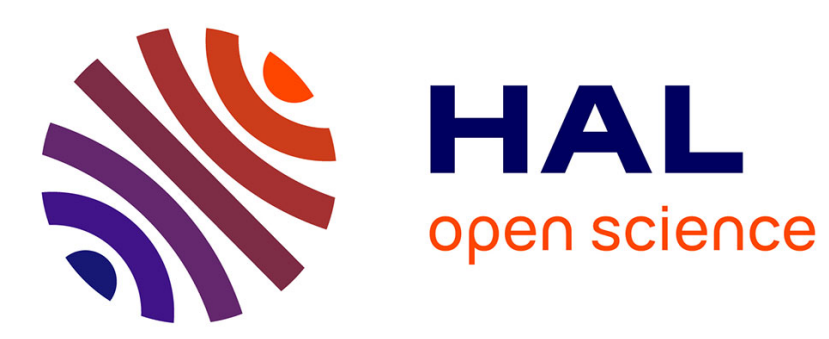

\title{
Auxiliary Variable-based Balancing (AVB) for source term treatment in open channel simulations
}

\author{
Carole Delenne, Vincent Guinot
}

\section{To cite this version:}

Carole Delenne, Vincent Guinot. Auxiliary Variable-based Balancing (AVB) for source term treatment in open channel simulations. Advances in Water Resources, 2012, 44, pp.85-100. 10.1016/j.advwatres.2012.05.007 . hal-01196833

\section{HAL Id: hal-01196833 \\ https://hal.science/hal-01196833}

Submitted on 10 Sep 2015

HAL is a multi-disciplinary open access archive for the deposit and dissemination of scientific research documents, whether they are published or not. The documents may come from teaching and research institutions in France or abroad, or from public or private research centers.
L'archive ouverte pluridisciplinaire HAL, est destinée au dépôt et à la diffusion de documents scientifiques de niveau recherche, publiés ou non, émanant des établissements d'enseignement et de recherche français ou étrangers, des laboratoires publics ou privés. 


\title{
Auxiliary Variable-based Balancing (AVB) for source term treatment in open channel simulations
}

\author{
Carole Delenne, Vincent Guinot \\ Université Montpellier 2, HydroSciences Montpellier UMR 5569 (CNRS, IRD, UM1, UM2), CC05\%, Place \\ Eugène Bataillon, 34095 Montpellier Cedex 5, France.
}

\section{- Abstract}

Practical engineering applications of open channel flow modelling involve geometric terms arising from variations in channel shape, bottom slope and friction. This paper presents the family of schemes that satisfy the generalised $C$-property for which static equilibrium is a particular case, in the framework of one-dimensional open channel flows. This approach, named Auxiliary Variable-based Balancing, consists of using an auxiliary variable in place of the flow variables in the diffusive part of the flux estimate. The auxiliary variable is defined so as to achieve a zero gradient under steady-state conditions, whatever the geometry. Many approaches presented in the litterature can be viewed as a particular AVB case. Three auxiliary variables are presented in this paper: water elevation, specific force and hydraulic head. The methodology is applied to three classical Riemann solvers: HLL, Roe and the $Q$-scheme. The results are compared on five test-cases: three steady-state configurations including friction, singular head losses and variations in bottom elevation, channel width and banks slope and two transient test-case (dam-break problems on rectangular and triangular channel). In each case, the auxiliary variable that best preserves the steady-state configuration is the hydraulic head. Besides, using the head as auxiliary variable allows head loss functions due to singularities to be incorporated directly in the governing equations, without the need for internal boundaries. However, it is generally less accurate when sharp transients are involved.

\section{Keywords}

Shallow water equations; Finite volume method; $C$-property; well-balancing; non-prismatic channel; trapezoidal channel; geometric source terms.

\section{Introduction}

In hydrodynamic modelling, real-world applications of computational open channel simulations involve the discretization of source terms arising from bottom slope, non-prismatic channel, etc. Attempting to discretize the fluxes and source terms independently from each other usually leads to stability problems. An indispensable prerequisite is that the discretization of flux gradients and geometric source terms should allow static equilibrium conditions to be preserved. This is known as the $C$-property $[4,38]$. The need for source term discretization techniques that preserve equilibrium conditions without introducing spurious oscillations in the computed variables has led to the general notion of well-balanced schemes. Over the past two decades, substantial research effort has been devoted to the influence of source terms discretization techniques [32] and new definitions that preserve the $C$-property, including applications to high-order schemes such as WENO (weighted essentially non-oscillatory) methods (e.g. [7, 10, 13, 39]).

The various existing source term discretization approaches may be classified into two broad families: (i) approaches where the source term discretization technique is adapted to the flux formulae, and (ii) approaches where the flux formulae are adapted from, or derived in a coupled way with, the source term discretization. Examples of the former approach are source term upwinding [4,38] and derived techniques such as predictor-corrector [3] or introduction of the source terms in the flux formulation [9], divergence form for the 
bed slope source term (DBF) [37], the quasi-steady wave propagation method [29], asymptotic balancing [12] or the source term projection technique in discontinuous Galerkin techniques [27]. Examples of the latter are the well-balanced approach [1, 2, 8, 24, 31, 35], flux and source term splitting [11], characteristics-based approximate-state and augmented Riemann solvers $[10,16,18,20,30]$, the homogeneous approach [28] and other static equilibrium-preserving techniques $[6,19,40]$.

Various solutions have also been proposed to enforce the $C$-property in finite volumebased discretizations. One of the earliest solutions, proposed in [33] for the solution of the SWE and later extended in [40], consists in replacing the water depth with the free surface elevation. This option can be extended to the open channel equations in arbitrary-shaped channels, as shown in the present paper. It has the drawback that simple flow configurations such as uniform flow over a constant slope cannot be computed accurately (see section 3.2). Another option is to approximate the variations in the cross-sectional area with a consistent estimate taken from the balance between the specific force and the source term in the momentum equation [6]. The estimate is defined in such a way that it is zero under steady state conditions. Very similar formulae to that of [6] have been obtained using completely different approaches in $[28,30]$. The approaches $[6,28,30]$ have the common point that the gradient in one of the flow variables is replaced with the gradient in another variable, called auxiliary variable hereafter. This gradient is zero under static conditions. That different approaches yield the same formulae lead to wonder whether a general methodology can be derived to define auxiliary variables.

In the present paper, the principle of Auxiliary Variable-based Balancing (AVB) is presented for one-dimensional free surface flow calculations in non-prismatic, trapezoidal channels. This is motivated by the fact that in industrial open channel packages, the cross-sectional geometry is broken into a set of trapezoidal elements. The AVB approach is used to derive flux formulae that allow non-static, steady state flow conditions to be preserved, even at low orders of discretization, that is, when first-order schemes are used.

The principle of the AVB method is presented for the water hammer and one-dimensional SWE in [25]. However, the one-dimensional shallow water equations are a very simplified description of free surface flows in natural channels. Besides, only one possible approach for source term discretization (a variant of source term upwinding) is considered in [25]. The applicability of the approach to more complex cross-sections and other source term discretization approaches is not investigated in [25]. The objectives of the present paper are (i) to present the methodology of Auxiliary Variable-based Balancing (AVB), (ii) to apply the AVB approach to the open channel flow equations in a well-balanced, finite volume framework, (iii) to provide the flux and source term discretizations for a variety of Riemann solvers, and (iv) to analyse the accuracy of the numerical solutions obtained using a number of various AVB-based discretizations. As mentioned above, first-order space discretizations are retained for the sake of computational rapidity.

The structure of the paper is as follows. Section 2 presents the governing equations and their discretisation. The AVB methodology is detailed in section 3 and its application to classical Riemann solvers presented in section 4. Section 5 provides computational examples, including steady-state configurations and transient test-cases as well as a convergence analysis for the classical dam-break problem (for which an analytical solution is available).

\section{Governing equations and solution method}

\subsection{Governing equations}

The purpose is to solve $2 \times 2$ hyperbolic systems of conservation laws in the form

$$
\frac{\partial \mathbf{U}}{\partial t}+\frac{\partial \mathbf{F}}{\partial x}=\mathbf{S}
$$

where $\mathbf{U}, \mathbf{F}$ and $\mathbf{S}$ are defined as

$$
\mathbf{U}=\left[\begin{array}{c}
A \\
Q
\end{array}\right], \mathbf{F}=\left[\begin{array}{c}
Q \\
M
\end{array}\right]=\left[\begin{array}{c}
Q \\
\frac{Q^{2}}{A}+\frac{P}{\rho}
\end{array}\right], \mathbf{S}=\left[\begin{array}{c}
0 \\
\left(S_{0}-S_{f}\right) g A+\frac{R}{\rho}
\end{array}\right]
$$



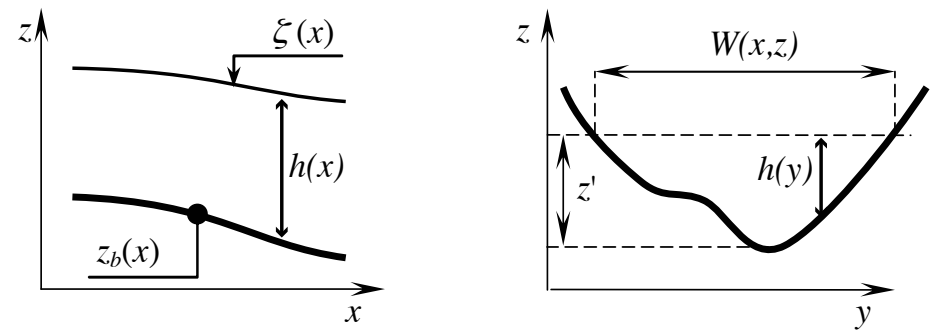

Figure 1: Channel geometry. Left) longitudinal view:bottom and water elevation. Right) transversal view: channel width and depth.

the bottom and energy slope, $R$ is the $x$-component of the reaction of the walls onto the water (if the channel is non-prismatic) and $\rho$ is the water density.

The forces $P$ and $R$ are derived from the assumption of a hydrostatic pressure distribution and obey the following definitions [14]:

$$
\begin{aligned}
& \frac{P}{\rho}=\int_{A}(\zeta-z) g \mathrm{~d} A=\int_{0}^{h}\left(h-z^{\prime}\right) g W\left(z^{\prime}\right) \mathrm{d} z^{\prime} \\
& \frac{R}{\rho}=\int_{0}^{h}\left(h-z^{\prime}\right) g\left(\frac{\partial W}{\partial x}\right)_{h-z^{\prime}=\text { Const }}\left(z^{\prime}\right) \mathrm{d} z^{\prime}
\end{aligned}
$$

where $W(z)$ is the width of the channel at the elevation $z, h$ is the water depth (that is the distance between the lowest point in the cross-section and the free surface), $z^{\prime}=z-z_{b}$ is the elevation above the bottom lowest point and $\zeta$ is the free surface elevation (Figure 1).

The energy slope is classically assumed to obey a turbulent-type friction law such as Manning's law:

$$
S_{f}=n_{M}^{2} u^{2} R_{H}^{-4 / 3}
$$

where $n_{M}$ is Manning's friction coefficient, $u=Q / A$ is the flow velocity and $R_{H}$ is the hydraulic radius, defined as the ratio of the cross-sectional area $A$ to the wetted perimeter $\chi$, yielding

$$
S_{f}=n_{M}^{2} Q^{2} A^{-10 / 3} \chi^{4 / 3}
$$

It is noted that the Jacobian matrix $\mathbf{A}$ of $\mathbf{F}$ with respect to $\mathbf{U}$ is given by

$$
\mathbf{A}=\frac{\partial \mathbf{F}}{\partial \mathbf{U}}=\left[\begin{array}{cc}
0 & 1 \\
c^{2}-u^{2} & 2 u
\end{array}\right]
$$

111

where the speed $c$ of the waves in still water is defined as

$$
c^{2} \equiv \frac{\partial\left(\frac{P}{\rho}\right)}{\partial A}=\frac{g A}{b}
$$

112 where $b=W(\zeta)$ is the top width of the channel. The matrix $\mathbf{A}$ can be diagonalized into a 113 matrix $\boldsymbol{\Lambda}$ defined as:

$$
\begin{gathered}
\boldsymbol{\Lambda}=\left[\begin{array}{cc}
\lambda^{(1)} & 0 \\
0 & \lambda^{(2)}
\end{array}\right] \\
\lambda^{(1)}=u-c \\
\lambda^{(2)}=u+c
\end{gathered}
$$

114 The problem is assumed to be properly posed hereafter, that is, the initial and boundary 115 conditions are specified such that Eq. (1) can be solved uniquely for $\mathbf{U}$ at all points of a 116 computational domain $[0, L]$ for all times $t>0$. 


\subsection{Finite volume discretization}

Eq. (1) is discretized using a finite volume formalism as

$$
\mathbf{U}_{i}^{n+1}=\mathbf{U}_{i}^{n}+\frac{\Delta t}{\Delta x_{i}}\left(\mathbf{F}_{i-\frac{1}{2}}^{n+\frac{1}{2}}-\mathbf{F}_{i+\frac{1}{2}}^{n+\frac{1}{2}}\right)+\Delta t \mathbf{S}_{i}^{n+\frac{1}{2}}
$$

where the subscript $i$ denotes a cell average, subscripts $i \pm \frac{1}{2}$ denote estimates at the interfaces between the computational cells, the superscript $n$ indicates that the variable is estimated a time level $n$, and the superscript $n+\frac{1}{2}$ denotes an average value between time levels $n$ and $n+1$, and where $\Delta x_{i}$ is the width of he computational cell $i$. In explicit schemes, the variables with superscripts $n+\frac{1}{2}$ are computed using the known values at the time level $n$; in implicit schemes, the unknown values at the time level $n+1$ are used.

In what follows, non-prismatic, trapezoidal cross-sections are considered. The reason for this is that in all commercial open channel packages, the channel geometry is discretized into a series of trapezia. Consequently, the capability to deal with trapezoidal cross-sections is seen as an indispensable prerequisite to a generalisation of the method to arbitrary-shaped channels. Note that rectangular and triangular cross-sections are obtained as particular cases of the proposed approach, as illustrated by a number of computational examples in Section 5.

The geometric parameters of the cross-sections are defined at the interfaces between the computational cells. They are interpolated linearly within the cells. Consequently, the geometry is continuous at the cell interfaces. Assuming non-prismatic trapezoidal channel geometry, the width $W(x, z)$ at a given abscissa $x$ and elevation $z$ takes the form

$$
W(x, z)=W_{0}(x)+\left(z-z_{b}(x)\right) W_{1}(x)
$$

where $W_{0}(x)$ is the bottom width of the channel at the abscissa $x, W_{1}(x)$ is the derivative of $W$ with respect to $z$ and $z_{b}$ is the bed elevation at the abscissa $x$. As mentioned above, $W_{0}, W_{1}$ and $z_{b}$ are assumed to vary linearly with $x$ within the cells. In the cell $i$, one has:

$$
\begin{gathered}
W_{0}(x)=W_{0, i-\frac{1}{2}}+\left(x-x_{i-\frac{1}{2}}\right) W_{0, i}^{(x)} \\
W_{1}(x)=W_{1, i-\frac{1}{2}}+\left(x-x_{i-\frac{1}{2}}\right) W_{1, i}^{(x)} \\
z_{b}(x)=z_{b, i-\frac{1}{2}}+\left(x-x_{i-\frac{1}{2}}\right) z_{b, i}^{(x)}
\end{gathered}
$$

where the superscript $(x)$ denotes the derivative with respect to $x$ :

$$
\begin{gathered}
W_{0, i}^{(x)}=\frac{W_{0, i+\frac{1}{2}}-W_{0, i-\frac{1}{2}}}{\Delta x_{i}} \\
W_{1, i}^{(x)}=\frac{W_{1, i+\frac{1}{2}}-W_{1, i-\frac{1}{2}}}{\Delta x_{i}} \\
z_{b, i}^{(x)}=\frac{z_{b, i+\frac{1}{2}}-z_{b, i-\frac{1}{2}}}{\Delta x_{i}}=S_{0, i}
\end{gathered}
$$

The cross-sectional area $A$ is given as the integral of $W$ between the bottom level and the free surface elevation:

$$
A(x)=\int_{z_{b}}^{\zeta} W(x, z) \mathrm{d} z=\left[W_{0}(x)+W_{1}(x) \frac{h(x)}{2}\right] h(x)
$$

142 143

where $h(x)$ is the water depth at the abscissa $x$. Assuming that the free surface is horizontal in the cell $i$ (which is true in the case of the first-order Godunov scheme), the average cell value $A$ is given by

$$
\begin{gathered}
A_{i}(\zeta) \equiv \frac{1}{\Delta x_{i}} \int_{x_{i-\frac{1}{2}}}^{x_{i+\frac{1}{2}}} A(x) \mathrm{d} x=\alpha_{i} h_{i-\frac{1}{2}}^{2}+\beta_{i} h_{i-\frac{1}{2}}+\gamma_{i} \\
h_{i-\frac{1}{2}}=\left(\zeta-z_{b}\right)_{i-\frac{1}{2}} \\
\alpha_{i}=\frac{1}{2} W_{1, i-\frac{1}{2}}+\frac{\Delta x_{i}}{4} W_{1, i}^{(x)}
\end{gathered}
$$


71 174 Eq. (10): Eq. (6):

$$
\begin{gathered}
\beta_{i}=W_{0, i-\frac{1}{2}}+\frac{\Delta x_{i}}{2}\left(W_{0, i}^{(x)}+S_{0, i} W_{1, i-\frac{1}{2}}\right)+\frac{\Delta x_{i}^{2}}{3} S_{0, i} W_{1, i}^{(x)} \\
\gamma_{i}=\frac{\Delta x_{i}}{2} S_{0, i} W_{0, i-\frac{1}{2}}+\frac{\Delta x_{i}^{2}}{6}\left(2 S_{0, i} W_{0, i}^{(x)}+S_{0, i}^{2} W_{1, i-\frac{1}{2}}\right)+\frac{\Delta x_{i}^{3}}{8} S_{0, i}^{2} W_{1, i}^{(x)}
\end{gathered}
$$

\subsection{Flux calculation}

Approximate Riemann solvers provide flux formulae that can be recast in the following form, derived from [17]:

$$
\mathbf{F}=a \mathbf{F}_{L}+(1-a) \mathbf{F}_{R}+\mathbf{D}\left(\mathbf{U}_{L}-\mathbf{U}_{R}\right)
$$

where $L$ and $R$ denote respectively the left and right states of the Riemann problem, $a$ is a coefficient between 0 and 1 and $\mathbf{D}$ is a diffusion matrix that contributes to stabilise the numerical solution. The left and right states are obtained from an appropriate reconstruction, the simplest possible option (the first-order Godunov scheme [23]) being to use the average cell values. For $a=1 / 2$, Eq. (20) is the sum of a centred flux and a so-called artificial viscosity term. Both $a$ and $\mathbf{D}$ are functions of the wave speeds, in other words, the eigenvalues of the Jacobian matrix $\mathbf{A}$ of $\mathbf{F}$ with respect to $\mathbf{U}$ (Eq. 9).

How the left and right states for the Riemann problem are to be computed from the average cell values is dealt with in Section 3.

\subsection{Source term discretization}

The momentum source term is discretized explicitly.

The friction source term is computed by applying explicit estimates to the terms in

$$
\begin{gathered}
\left(S_{f}\right)_{i}^{n}=n_{M}^{2}\left(u^{2} R_{H}^{4 / 3}\right)_{i}^{n}=\left(\frac{W_{0}+\frac{1}{2} W_{1} h}{W_{0}+h\left(W_{1}^{2}+4\right)^{1 / 2}}\right)_{i}^{n} \\
\left(S_{f}\right)_{i}^{n}=n_{M}^{2}\left(Q^{2} A^{2 / 3} \chi^{-4 / 3}\right)_{i}^{n}=n_{m}^{2}\left(Q^{2}\left(W_{0}+\frac{1}{2} W_{1} h\right)^{2 / 3}\left(W_{0}+h \sqrt{W_{1}^{2}+4}\right)^{-4 / 3}\right)_{i}^{n}
\end{gathered}
$$

The geometric source term $g A S_{0}+\frac{R}{\rho}$ is rather difficult to compute directly under the assumption of varying $W_{0}, W_{1}$ and $z_{b}$. However, it can be estimated cell-wise from simple balance considerations. Consider static equilibrium conditions, i.e., $Q=0$ in all cells, then Eqs. (1) and (10) yield

$$
\left(g A S_{0}+\frac{R}{\rho}\right)_{i}^{n} \Delta x_{i}=\left(\frac{P}{\rho}\right)_{i+\frac{1}{2}}^{n}-\left(\frac{P}{\rho}\right)_{i-\frac{1}{2}}^{n}
$$

Given the definition (Eq 11) of the channel width, and the specific pressure force $\frac{P}{\rho}$ (Eq. 3), one has

$$
\frac{P}{\rho}=g \int_{0}^{h}\left(h-z^{\prime}\right)\left(W_{0}(x)+z^{\prime} W_{1}(x)\right) \mathrm{d} z^{\prime}=\frac{1}{2} g W_{0}(x) h^{2}+\frac{1}{6} g W_{1}(x) h^{3}
$$

The value of the specific pressure force at the interface $i-\frac{1}{2}$ is then easily computedas

$$
\left(\frac{P}{\rho}\right)_{i-\frac{1}{2}}^{n}=\frac{g}{2} W_{0, i-\frac{1}{2}} h_{i-\frac{1}{2}}^{2}+\frac{g}{6} W_{1, i-\frac{1}{2}} h_{i-\frac{1}{2}}^{3}
$$

with the definition (19b) for $h_{i-\frac{1}{2}}$. Since the purpose is to estimate the source term in the cell $i$, the free surface elevation to be used in Eq. (19b) is $\zeta_{i}^{n}$. The same formulation can be obtained at interface $i+\frac{1}{2}$ yielding the final estimate for the source term to be used in

$$
\begin{aligned}
\left(g A S_{0}+\frac{R}{\rho}\right)_{i}^{n} \Delta x_{i}= & \frac{g}{2}\left(W_{0, i+\frac{1}{2}}+\frac{1}{3} W_{1, i+\frac{1}{2}} h_{i+\frac{1}{2}, i}\right) h_{i+\frac{1}{2}, i}^{2} \\
& -\frac{g}{2}\left(W_{0, i-\frac{1}{2}}+\frac{1}{3} W_{1, i-\frac{1}{2}} h_{i-\frac{1}{2}, i}\right) h_{i-\frac{1}{2}, i}^{2}
\end{aligned}
$$




$$
\begin{aligned}
h_{i-\frac{1}{2}, i} & =\zeta_{i}^{n}-z_{b, i-\frac{1}{2}} \\
h_{i+\frac{1}{2}, i} & =\zeta_{i}^{n}-z_{b, i+\frac{1}{2}}
\end{aligned}
$$

\subsection{Balancing issues}

The discretization of the source term in the momentum equation usually poses no problem. This issue has been dealt with abundantly in the literature, within a very wide variety of techniques $[1,2,4,6,27,28,38,40]$. A remaining problem encountered in practical applications is related to the continuity equation and the difference often observed between the average cell values and the interface values for the volume discharge.

Consider a solution $\mathbf{U}$ verifying steady state, $\frac{\partial \mathbf{U}}{\partial t}=0$. The first component of Eq. (1) imposes that $Q$ be equal to a constant $Q_{0}$ all throughout the computational domain. In particular, the discharge $Q$ computed at the cell interfaces should be identical to that in the cell values. This, however, is not necessarily the case if Eq. (20) is used. Indeed, writing the first component of Eq. (20) leads to the following formula for the interface flux

$$
Q_{0}=\left(a+D_{12}\right) Q_{L}+\left(1-a-D_{12}\right) Q_{R}+D_{11}\left(A_{L}-A_{R}\right)
$$

where $D_{11}$ and $D_{12}$ are the components on the first row of the artificial viscosity matrix $\mathbf{D}$. Eq. (27) can be rewritten as

$$
\left(a+D_{12}\right) Q_{L}+\left(1-a-D_{12}\right) Q_{R}=Q_{0}+D_{11}\left(A_{R}-A_{L}\right)
$$

Assume that the discretized solution has reached steady state. If the geometry of the channel is arbitrary (non-constant bottom slope and/or non-prismatic channel), in general $A_{L} \neq A_{R}$. It is then obvious from Eq. (28) that at least one of the discharges $Q_{L}$ and $Q_{R}$ is different from the uniform discharge $Q_{0}$. Consequently, a non-uniform discharge profile is obtained. In particular, if the initial situation is static $\left(Q_{0}=0\right)$, non-zero discharges are computed. Artificial oscillations appear and propagate throughout the computational domain.

The ability of a numerical scheme to preserve static equilibrium conditions has been introduced as the $C$-property in [4]. Specifying the $C$-property exactly or approximately has proved to lead to efficient source term balancing techniques. The most widespread approach consists in adapting the discretization of the source term to the formulation of the flux so as to satisfy the $C$-property. In the Auxiliary Variable-based Balancing (AVB) approach, the opposite approach is followed: the formulation of the flux gradients is adapted to that of the source term.

\section{Auxiliary Variable-based Balancing method}

\subsection{Principle}

The AVB method is based on the following requirements: (i) the artificial viscosity term in Eq. (20) should be modified in such a way that diffusion becomes zero when steady state is reached; (ii) the source term in the momentum equation should be discretized in such a way that it does not influence the calculation of the flux in the intermediate region of constant state. The second issue has been addressed in subsection 2.4 (source term discretization); the first issue is dealt with in the following subsections.

AVB uses an auxiliary variable $\mathbf{V}$ in the expression of the artificial viscosity term:

$$
\mathbf{F}=a \mathbf{F}_{L}+(1-a) \mathbf{F}_{R}+\mathbf{D}_{\mathbf{V}}\left(\mathbf{V}_{L}-\mathbf{V}_{R}\right)
$$

where $\mathbf{V}$ is a function of both the variable $\mathbf{U}$ and the parameter $\varphi, \mathbf{V}=\mathbf{V}(\mathbf{U}, \varphi)$. The diffusion matrix $\mathbf{D}_{\mathbf{V}}$ and the auxiliary variable $\mathbf{V}$ are chosen such that the following conditions are verified:

(C1): under steady state conditions, $\mathbf{V}_{L}=\mathbf{V}_{R}$.

(C2): for $\varphi=$ Const, $\mathbf{D}_{\mathbf{V}}\left(\mathbf{V}_{L}-\mathbf{V}_{R}\right)=\mathbf{D}\left(\mathbf{U}_{L}-\mathbf{U}_{R}\right)$ 
with

$$
\begin{gathered}
\mathbf{D}_{\mathbf{V}}\left(\mathbf{V}_{L}-\mathbf{V}_{R}\right)=-\boldsymbol{\Delta} x \mathbf{D}_{\mathbf{V}} \frac{\partial \mathbf{V}}{\partial x}+\operatorname{HOT}(\Delta x) \\
\mathbf{D}\left(\mathbf{U}_{L}-\mathbf{U}_{R}\right)=-\boldsymbol{\Delta} x \mathbf{D} \frac{\partial \mathbf{U}}{\partial x}+\operatorname{HOT}(\Delta x)
\end{gathered}
$$

$$
\operatorname{HOT}(\Delta x) \underset{\Delta x \rightarrow 0}{\rightarrow} 0
$$

Noticing that $\mathbf{V}=\mathbf{V}(\mathbf{U}, \varphi)$, the derivative of $\mathbf{V}$ with respect to $x$ is expressed as

$$
\frac{\partial \mathbf{V}}{\partial x}=\frac{\partial \mathbf{V}}{\partial \mathbf{U}} \frac{\partial \mathbf{U}}{\partial x}+\frac{\partial \mathbf{V}}{\partial \varphi} \frac{\partial \varphi}{\partial x}
$$

Consequently, $\mathbf{D}_{\mathbf{V}}$ is given by

$$
\mathbf{D}_{\mathbf{V}}=\mathbf{D}\left(\frac{\partial \mathbf{V}}{\partial \mathbf{U}}\right)^{-1}
$$

\subsection{Balancing option 1: free surface elevation}

One of the earliest examples of the use of an auxiliary variable is found in [33] for the solution of the shallow water equations where the free surface elevation $\zeta=z_{b}+h$ is used in place of the water depth $h$. The rationale is that under static conditions, the free surface elevation is constant, consequently, both $\mathrm{d} \zeta$ and $\mathrm{d} Q$ are zero at equilibrium. Note that the approach has been extended to the reconstruction technique in higher-order schemes in [40]. This leads to the following possible definition for the auxiliary variable

$$
\mathrm{d} \mathbf{V}_{\mathbf{1}}=\left[\begin{array}{c}
\mathrm{d} \zeta \\
\mathrm{d} Q
\end{array}\right]
$$

Since $\mathrm{d} A=b \mathrm{~d} \zeta$, one has from the definition of $\mathbf{U}$ in Eq. (2):

$$
\frac{\partial \mathbf{V}_{\mathbf{1}}}{\partial \mathbf{U}}=\left[\begin{array}{cc}
b^{-1} & 0 \\
0 & 1
\end{array}\right],\left(\frac{\partial \mathbf{V}_{\mathbf{1}}}{\partial \mathbf{U}}\right)^{-1}=\left[\begin{array}{ll}
b & 0 \\
0 & 1
\end{array}\right]
$$

This leads to the following artificial viscosity term:

$$
\mathbf{D}_{\mathbf{V}}\left(\mathbf{V}_{1 L}-\mathbf{V}_{\mathbf{1} R}\right)=\mathbf{D}\left[\begin{array}{cc}
b & 0 \\
0 & 1
\end{array}\right]\left[\begin{array}{c}
\zeta_{L}-\zeta_{R} \\
Q_{L}-Q_{R}
\end{array}\right]=\mathbf{D}\left[\begin{array}{c}
\left(\zeta_{L}-\zeta_{R}\right) b \\
Q_{L}-Q_{R}
\end{array}\right]
$$

Note that in the case of the SWE, $b=1$ and Nujic's [33] approach is retrieved.

This option has the drawback that steady state, uniform flow cannot be maintained exactly. Indeed, under uniform flow conditions, $Q_{L}=Q_{R}$ but the free surface elevations in two adjacent cells are not identical, $\zeta_{L} \neq \zeta_{R}$. Therefore, the artificial diffusion term in the continuity equation is non-zero and the interface flux is not equal to $Q_{L}=Q_{R}$. This is substantiated by the computational examples in Section 5 . 
3.3. Balancing option 2: specific force

This is the option explored in [6]. Similar formulae were obtained for the one-dimensional shallow water equations in [28, 30, 18], albeit from different considerations. The latter three approaches, however, focus on rectangular channels, while the proposed approach is applicable to arbitrary-shaped channels.

The specific force is used in place of the cross-sectional area in the first component of the auxiliary variable $\mathbf{V}$. The motivation is that under dynamic equilibrium (that is, under steady state flow conditions), the variations in the specific force are balanced exactly by the source terms in the momentum equation noted $S_{M}$. This leads to define the auxiliary variable $\mathbf{V}$ in differential form as

$$
\mathrm{d} \mathbf{V}=\left[\begin{array}{c}
\mathrm{d} M-S_{M} \\
\mathrm{~d} Q
\end{array}\right]
$$

254

Since $\mathrm{d} M=\left(c^{2}-u^{2}\right) \mathrm{d} A+2 u \mathrm{~d} Q$, one has

$$
\frac{\partial \mathbf{V}}{\partial \mathbf{U}}=\left[\begin{array}{cc}
c^{2}-u^{2} & 2 u \\
0 & 1
\end{array}\right],\left(\frac{\partial \mathbf{V}}{\partial \mathbf{U}}\right)^{-1}=\left[\begin{array}{cc}
\frac{1}{c^{2}-u^{2}} & -\frac{2 u}{c^{2}-u^{2}} \\
0 & 1
\end{array}\right]
$$

1 This leads to

$$
\frac{\partial \mathbf{V}}{\partial \mathbf{U}}=\left[\begin{array}{cc}
c^{2}-u^{2} & 0 \\
0 & 1
\end{array}\right],\left(\frac{\partial \mathbf{V}}{\partial \mathbf{U}}\right)^{-1}=\left[\begin{array}{cc}
\frac{1}{c^{2}-u^{2}} & 0 \\
0 & 1
\end{array}\right]
$$

262 and the following artificial viscosity term is obtained

$$
\mathbf{D}_{\mathbf{V}}\left(\mathbf{V}_{L}-\mathbf{V}_{R}\right)=\mathbf{D}\left[\begin{array}{c}
\frac{M_{L}-M_{R}-S_{M} \Delta x}{c^{2}-u^{2}} \\
Q_{L}-Q_{R}
\end{array}\right]
$$

263 This expression, however, remains invalid at critical points, for which $c^{2}=u^{2}$. As ${ }_{264}$ proposed in [6], in the case of 1D SWEs on rectangular channel, the final estimate for $\mathrm{d} \mathbf{V}$ is the minmod of the estimates given by the specific force option and the original approach:

$$
\mathrm{d} \mathbf{V}_{\mathbf{2}}=\operatorname{minmod}(\mathrm{d} \mathbf{V}, \mathrm{d} \mathbf{U})
$$

266

where $\mathrm{d} \mathbf{V}$ is defined by Eq. (42) and the minmod operator by:

$$
\operatorname{minmod}(a, b)= \begin{cases}\min (|a|,|b|) & \text { if } a b \geq 0 \\ 0 & \text { if } a b<0\end{cases}
$$

\subsection{Balancing option 3: hydraulic head}

In this option, presented in [25] (for 1D SWEs on rectangular channel), the hydraulic head $H=\zeta+\frac{u^{2}}{2 g}$ is used as auxiliary variable:

$$
\mathrm{d} \mathbf{V}=\left[\begin{array}{c}
\mathrm{d} H-S_{f} \\
\mathrm{~d} Q
\end{array}\right]
$$

Since $\mathrm{d} H=\left(\frac{1}{b}-\frac{u^{2}}{g A}\right) \mathrm{d} A+\frac{u}{g A} \mathrm{~d} Q=\frac{1}{b}\left[\left(1-F^{2}\right) \mathrm{d} A+\frac{F}{c} \mathrm{~d} Q\right]$, one has

$$
\frac{\partial \mathbf{V}}{\partial \mathbf{U}}=\left[\begin{array}{cc}
\frac{1-F^{2}}{b} & \frac{F}{b c} \\
0 & 1
\end{array}\right],\left(\frac{\partial \mathbf{V}}{\partial \mathbf{U}}\right)^{-1}=\left[\begin{array}{cc}
\frac{b}{1-F^{2}} & -\frac{\frac{F}{c}}{1-F^{2}} \\
0 & 1
\end{array}\right]
$$


${ }_{271}$ This leads to the following artificial viscosity term:

$$
\mathbf{D}_{\mathbf{V}}\left(\mathbf{V}_{L}-\mathbf{V}_{R}\right)=\mathbf{D}\left[\begin{array}{c}
\frac{\left(H_{L}-H_{R}-S_{f} \Delta x\right) b-\frac{F}{c}\left(Q_{L}-Q_{R}\right)}{1-F^{2}} \\
Q_{L}-Q_{R}
\end{array}\right]
$$

272 For the same reasons as Option 2, the following variation is proposed for Option 3:

$$
\mathrm{d} \mathbf{V}=\left[\begin{array}{c}
\mathrm{d} H-S_{f}-\frac{F}{c} \mathrm{~d} Q \\
\mathrm{~d} Q
\end{array}\right]
$$

${ }_{273}$ The Jacobian matrix of $\mathbf{V}$ with respect to $\mathbf{U}$ is given by:

$$
\frac{\partial \mathbf{V}}{\partial \mathbf{U}}=\left[\begin{array}{cc}
\frac{1-F^{2}}{b} & 0 \\
0 & 1
\end{array}\right],\left(\frac{\partial \mathbf{V}}{\partial \mathbf{U}}\right)^{-1}=\left[\begin{array}{cc}
\frac{b}{1-F^{2}} & 0 \\
0 & 1
\end{array}\right]
$$

${ }_{274}$ This leads to the following artificial viscosity term:

$$
\mathbf{D}_{\mathbf{V}}\left(\mathbf{V}_{L}-\mathbf{V}_{R}\right)=\mathbf{D}\left[\begin{array}{c}
\frac{H_{L}-H_{R}-S_{f} \Delta x}{1-F^{2}} \\
Q_{L}-Q_{R}
\end{array}\right]
$$

275 As in option 2, this expression is not valid at critical points for which $F=1$, the final 276 estimate for $\mathrm{d} \mathbf{V}$ is thus

$$
\mathrm{d} \mathbf{V}_{\mathbf{3}}=\operatorname{minmod}(\mathrm{d} \mathbf{V}, \mathrm{d} \mathbf{U})
$$

277 where $\mathrm{d} \mathbf{V}$ is defined by Eq. (50).

278 Note that if a singular head loss $\Delta H_{s}$ is to be introduced, it can also be taken into 279 account in the artificial viscosity term:

$$
\mathbf{D}_{\mathbf{V}}\left(\mathbf{V}_{L}-\mathbf{V}_{R}\right)=\mathbf{D}\left[\begin{array}{c}
\frac{H_{L}-H_{R}-\Delta H_{s}-S_{f} \Delta x}{1-F^{2}} \\
Q_{L}-Q_{R}
\end{array}\right]
$$

282

The HLL solver [26] can be written in the form (20) by defining $a$ and D as

$$
\begin{gathered}
a=\frac{\lambda^{+}}{\lambda^{+}-\lambda^{-}} \\
\mathbf{D}=-\frac{\lambda^{-} \lambda^{+}}{\lambda^{+}-\lambda^{-}} \mathbf{I}
\end{gathered}
$$

${ }_{283}$ where $\mathbf{I}$ is the identity matrix and $\lambda^{-}, \lambda^{+}$are respectively estimates of the fastest waves ${ }_{284} \lambda^{(1)}$ and $\lambda^{(2)}$ defined in Eqs.. (9b, 9c) in the direction of negative and positive $x$ [15, 17]:

285

$$
\begin{aligned}
& \lambda^{-}=\min \left(u_{L}-c_{L}, u_{R}-c_{R}, 0\right) \\
& \lambda^{+}=\max \left(u_{L}+c_{L}, u_{R}+c_{R}, 0\right)
\end{aligned}
$$

286 4.2. Application to Roe's solver

${ }_{287}$ Roe's solver [34] can be written in the form (20) by setting

$$
\begin{gathered}
a=\frac{1}{2} \\
\mathbf{D}=\frac{\tilde{\mathbf{A}}^{ \pm}}{2}
\end{gathered}
$$

${ }_{288}$ where $\tilde{\mathbf{A}}^{ \pm}$is the matrix generated by the absolute values of the eigenvalues of $\mathbf{A}$ :

$$
\tilde{\mathbf{A}}^{ \pm}=\tilde{\mathbf{K}}|\tilde{\mathbf{\Lambda}}| \tilde{\mathbf{K}}^{-1}
$$

289 with

$$
\tilde{\mathbf{K}}=\left[\begin{array}{cc}
1 & 1 \\
\tilde{\lambda}^{(1)} & \tilde{\lambda}^{(2)}
\end{array}\right], \tilde{\mathbf{K}}^{-\mathbf{1}}=\frac{1}{\lambda^{(2)}-\lambda^{(1)}}\left[\begin{array}{cc}
\tilde{\lambda}^{(2)} & -1 \\
-\tilde{\lambda}^{(1)} & 1
\end{array}\right],|\tilde{\Lambda}|=\left[\begin{array}{cc}
\left|\tilde{\lambda}^{(1)}\right| & 0 \\
0 & \left|\tilde{\lambda}^{(2)}\right|
\end{array}\right]
$$


leading to the following expression for $\tilde{\mathbf{A}}^{ \pm}$:

$$
\begin{gathered}
\tilde{\mathbf{A}}^{ \pm}=\left[\begin{array}{ll}
a_{11} & a_{12} \\
a_{21} & a_{22}
\end{array}\right] \\
a_{11}=\frac{\tilde{\lambda}^{(2)}\left|\tilde{\lambda}^{(1)}\right|-\tilde{\lambda}^{(1)}\left|\tilde{\lambda}^{(2)}\right|}{\tilde{\lambda}^{(2)}-\tilde{\lambda}^{(1)}} \\
a_{12}=\frac{\left|\tilde{\lambda}^{(2)}\right|-\left|\tilde{\lambda}^{(1)}\right|}{\tilde{\lambda}^{(2)}-\tilde{\lambda}^{(1)}} \\
a_{21}=-\tilde{\lambda}^{(1)} \tilde{\lambda}^{(2)} a_{12} \\
a_{22}=\frac{\tilde{\lambda}^{(2)}\left|\tilde{\lambda}^{(2)}\right|-\tilde{\lambda}^{(1)}\left|\tilde{\lambda}^{(1)}\right|}{\tilde{\lambda}^{(2)}-\tilde{\lambda}^{(1)}}
\end{gathered}
$$

298

In Roe's approach [34], the eigenvalues $\tilde{\lambda}^{(1)}=(\tilde{u}-\tilde{c})$ and $\tilde{\lambda}^{(2)}=(\tilde{u}+\tilde{c})$ in the diagonal matrix $\widetilde{\boldsymbol{\Lambda}}$ are obtained from Roe's averages $[21,22]$ :

$$
\begin{gathered}
\widetilde{c}=\left[\frac{g}{2}\left(\frac{A_{L}}{b_{L}}+\frac{A_{R}}{b_{R}}\right)\right]^{1 / 2} \\
\widetilde{u}=\frac{c_{L} u_{L}+c_{R} u_{R}}{c_{L}+c_{R}}
\end{gathered}
$$

\subsection{Application to the $Q$-scheme}

The $Q$-scheme uses the same formula as Roe's formula, except that the matrix $\widetilde{\mathbf{A}}$ in Eq. (58) is estimated from the average of the left and right-hand cells

$$
\widetilde{\mathbf{A}}=\widetilde{\mathbf{A}}\left(\frac{\mathbf{U}_{L}+\mathbf{U}_{R}}{2}\right)
$$

yielding to the following approximation for the eigenvalues:

$$
\widetilde{c}=g\left(\frac{A_{L R}}{\tilde{b}}\right)^{1 / 2}
$$

$$
\widetilde{u}=\frac{Q_{L R}}{A_{L R}}
$$

where $\tilde{b}=W_{0, i-\frac{1}{2}}+h_{L R} W_{1, i-\frac{1}{2}}$ and $X_{L R}=\left(X_{L}+X_{R}\right) / 2(X \in\{A, Q, h\})$.

\subsection{Summary of formulae - Algorithmic aspects}

From an algorithmic point of view, the steps in the solution process are the following:

1. For each cell, compute the free surface elevations $\zeta_{L}$ and $\zeta_{R}$ from the left and right states $\mathbf{U}_{L}$ and $\mathbf{U}_{R}$, using the correspondence between $A$ and $h, \mathrm{Eq}(19 \mathrm{a})$. Use the free surface elevations to compute the geometric source term from Eqs (26).

2. For each interface, compute the flux $\mathbf{F}$ using Eq (29) with the fluxes on both sides of the interface $\left(\mathbf{F}_{L}, \mathbf{F}_{R}\right)$, the auxiliary variables $\mathbf{V}_{L}, \mathbf{V}_{R}$ according to the AVB option chosen and with $a$ depending on the solver.

3. Compute the friction source term as in Eq (21).

4. Apply the balance equation (10) to compute the hydrodynamic variable at the next time step. 


\begin{tabular}{lll}
\hline Symbol & Meaning & Value \\
\hline$g$ & Gravitational acceleration & $9.81 \mathrm{~m} \mathrm{~s}^{-2}$ \\
$L$ & Length of the domain & $3,000 \mathrm{~m}$ \\
$S_{0}$ & Bottom slope & $10^{-3}$ \\
$W_{0}$ & Channel width & $1 \mathrm{~m}$ \\
$W_{1}$ & Derivative of the width with respect to $z$ & 0 \\
$h_{0}$ & Initial water depth & $1 \mathrm{~m}$ \\
$z_{\mathrm{ds}}$ & Prescribed surface elevation downstream & $1 \mathrm{~m}$ \\
$Q_{\mathrm{up}}$ & Prescribed discharge upstream & $1 \mathrm{~m}^{3} \mathrm{~s}^{-1}$ \\
$n_{M}$ & Manning's friction coefficient & $0.025 \mathrm{~m}^{-1 / 3} \mathrm{~s}$ \\
$\Delta x$ & Computational cell width & $1 \mathrm{~m}$ \\
$\Delta t$ & Simulation time & $20,000 \mathrm{~s}$ \\
\hline
\end{tabular}

Table 1: Test 1 - steady state flow in a prismatic, rectangular channel. Parameters of the test case.
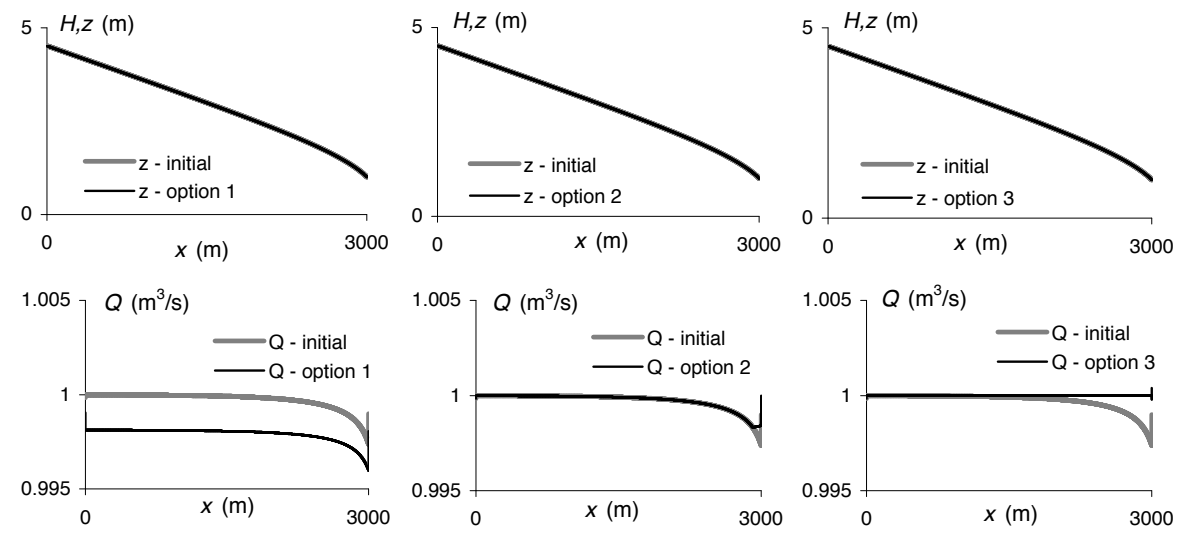

Figure 2: Test 1 - steady state flow in a prismatic, rectangular channel. Top: water elevation $z$ and hydraulic head $H$, down: discharge $Q$ obtained with $\mathbf{V}=\mathbf{U}$ (Initial) and with the three different AVB options, using the HLL solver (the results obtained with the Roe's and the $Q$-scheme solvers are identical).

\section{Computational examples}

\subsection{Steady state configurations}

5.1.1. Test 1: steady state flow in a prismatic, rectangular channel

In this test case, the various AVB options are applied to steady state flow in a prismatic, rectangular channel (i.e. with a constant value of $W_{0}$ and with $W_{1}=0$ ) including friction. A transient simulation is carried out from an initial state at rest until steady state is obtained. The parameters of the test case are given in Table 1.

Figure 2 shows the results obtained from the initial formulation i.e. with $\mathbf{V}=\mathbf{U}$ and with the three different AVB options. Only the HLL solver is shown in this case because the results obtained with the two other solvers are identical. The profiles of the free surface elevation $z$ and the hydraulic head $H$ are identical regardless of the AVB option used (note that the water elevation and the hydraulic head are nearly identical because of a small velocity).

However, under steady-state conditions, the discharge $Q$ is expected to be uniform over the entire domain and equal to $Q_{\text {up }}$. The only option that provides the correct value of $Q$ over the whole domain but the downstream boundary, is the third one, i.e. based on the hydraulic head.

Figure 3 shows results of the same test case but with the introduction of a singular head loss in the middle of the channel. The head loss is computed using a classical Borda relationship:

$$
\Delta H_{s}=\alpha \frac{v^{2}}{2 g}
$$

where $\alpha$ is arbitrarily chosen to $\alpha=5$ in this case, but can be estimated from any empirical law. Figure 3a presents results using HLL solver. Each option provides a good estimate of 

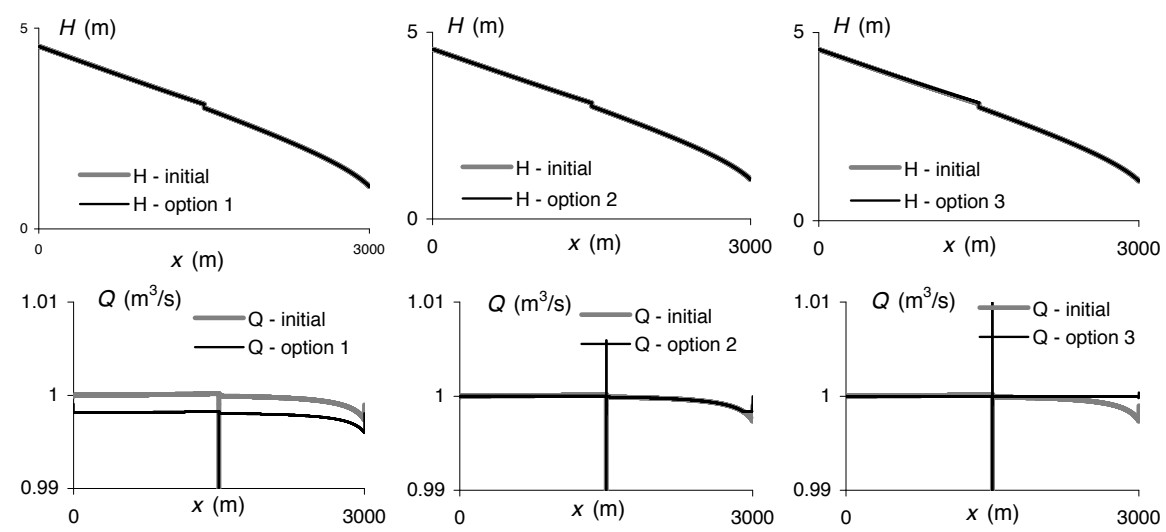

(a)
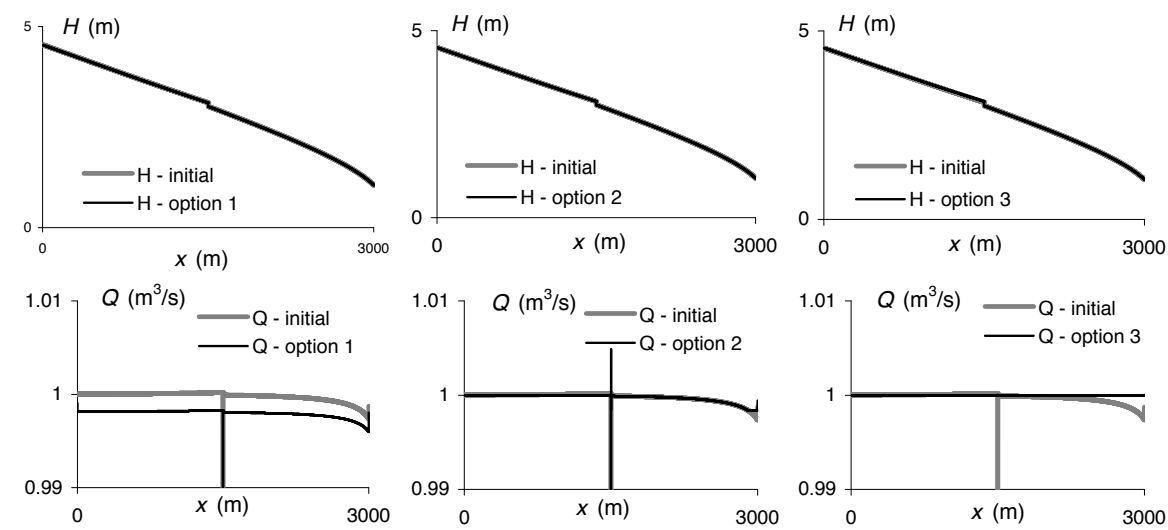

(b)

Figure 3: Test $1 \mathrm{~b}$ - steady state flow in a prismatic, rectangular channel with an arbitrary singular head loss in the middle of the channel. Top: water elevation $z$ and hydraulic head $H$, down: discharge $Q$ obtained with $\mathbf{V}=\mathbf{U}$ (Initial) and with the three different AVB options, using a) HLL solver, b) Roe's solver (identical to $Q$-scheme).

the hydraulic head and water elevation. In addition to the behaviour previously observed, the singular head loss triggers a spike in the discharge profiles when the HLL solver is used. Option 3, that explicitly takes into account the singular head loss in the flux computation, is the only one that provides a constant value $Q=Q_{\text {up }}$ with Roe's and $Q$-scheme solvers (Figure 3b).

\subsubsection{Test 2: frictionless steady state flow in a non-prismatic, rectangular channel}

The channel profile is shown in Figure 4; it contains two consecutive narrowings of the cross-section: the first one due to the width narrowing (minimum width at $25 \%$ of the channel length) and the second one to a bump in the bottom elevation (maximum elevation at $75 \%$ of the channel length). This is a frictionless test case, the parameters of which are given in Table 2. As for the first test case, a transient simulation is run for a sufficiently long time, so that the transient regime vanishes and steady state is reached. The prescribed discharge and downstream water level are chosen such that the flow regime is subcritical upstream of both the narrowing and the bump, yielding two hydraulic jumps.

Figure 5 shows results obtained with the different AVB options and the three solvers (note that the results obtained with Roe's solver and $Q$-scheme are identical). The profiles obtained for the hydraulic head $H$ and water elevation $z$ with the different solvers and options bear similarities except for the points upstream the channel narrowing. In constrast, substantial differences can be observed for the discharge $Q$. As for the first test case, the steady state configuration theoretically implies a constant value for the discharge. It can be seen that the same profile is obtained using the initial formulation (i.e. $\mathbf{V}=\mathrm{U}$ ) for the three solvers, and that this profile is the most different from the constant value of $Q=Q_{\text {up }}$. Option 2 also give a strongly variable discharge in space when used with HLLC, but not with Roe's solver or $Q$-scheme. Option 3 gives better results: it is very close to $Q=Q_{\text {up }}$ 

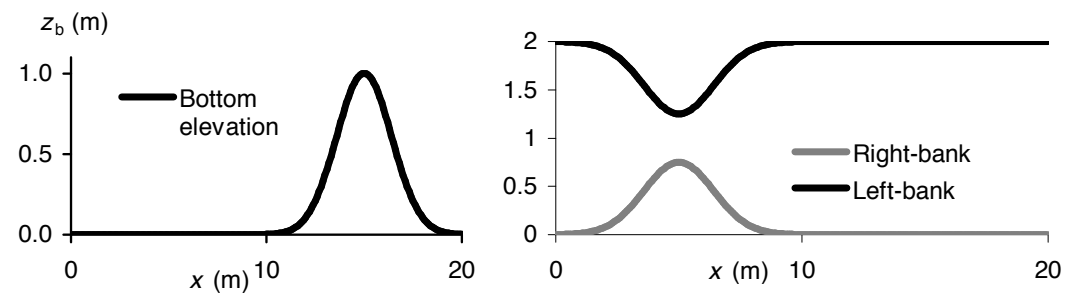

Figure 4: Test 2 - Frictionless steady state flow in a non-prismatic, rectangular channel. Channel profile: left, bottom elevation; right, left- and right-bank profile.

\begin{tabular}{lll}
\hline Symbol & Meaning & Value \\
\hline$g$ & Gravitational acceleration & $9.81 \mathrm{~m} \mathrm{~s}^{-2}$ \\
$L$ & Length of the domain & $20 \mathrm{~m}$ \\
$W_{0}, z_{b}$ & Channel width and bottom elevation & Figure 4 \\
$W_{1}$ & Derivative of the width with respect to $z$ & 0 \\
$z_{0}$ & Initial free surface elevation & $1.1 \mathrm{~m}$ \\
$z_{\mathrm{ds}}$ & Prescribed surface elevation downstream & $1.1 \mathrm{~m}$ \\
$Q_{\mathrm{up}}$ & Prescribed discharge upstream & $2 \mathrm{~m}^{3} \mathrm{~s}^{-1}$ \\
$n_{M}$ & Manning's friction coefficient & $0 \mathrm{~m}^{-1 / 3} \mathrm{~s}$ \\
$\Delta x$ & Computational cell width & $0.1 \mathrm{~m}$ \\
$\Delta t$ & Simulation time & $400 \mathrm{~s}$ \\
\hline
\end{tabular}

Table 2: Test 2 - Frictionless steady state flow in a non-prismatic, rectangular channel. Parameters of the test case.

over the whole domain except in the immediate vicinity of the hydraulic jump $(x \approx 17 \mathrm{~m})$.

\subsubsection{Test 3: frictionless steady-state flow in a non-prismatic trapezoidal channel}

The channel profile is shown in Figure 6. It presents two simultaneous reductions of the cross-section (bump and width narrowing), located at the same abscissa. The channel is not prismatic with a variable bank slope yielding a transition from a trapezoidal shape at the boundaries to a rectangular shape at half length. The parameters used for this steady-state, frictionless test case are given in Table 3.

The simulated free surface elevation $z$, hydraulic head $H$ and discharge $Q$, obtained with the three AVB options and the three solvers are given in Figure 7 . In this case again, all three AVB options provide improved solutions compared to that given by the initial formulation, for which the transition from subcritical to supercritical conditions (and viceversa) is observed to induce strong variations in the estimation of the discharge. This statement however is to be moderated concerning Option 2 combined with HLLC solver that also yields such variations. In a largely lesser extent, option 1 also exhibits some small variations in the discharge. Moreover, it can be seen that the abscissa of the hydraulic jump is not exactly located using Option 1 with Roe's solver or $Q$-scheme, with an increase in hydraulic head upstream the jump.

\begin{tabular}{lll}
\hline Symbol & Meaning & Value \\
\hline$g$ & Gravitational acceleration & $9.81 \mathrm{~m} \mathrm{~s}^{-2}$ \\
$L$ & Length of the domain & $20 \mathrm{~m}$ \\
$W_{0}, W_{1}$ & Channel width and its derivative with respect to $z$ & Figure 6 \\
$z_{b}$ & Bottom elevation & Figure 6 \\
$z_{0}$ & Initial free surface elevation & $1.2 \mathrm{~m}$ \\
$z_{\mathrm{ds}}$ & Prescribed surface elevation downstream & $1.2 \mathrm{~m}$ \\
$Q_{\mathrm{up}}$ & Prescribed discharge upstream & $4 \mathrm{~m}^{3} \mathrm{~s}^{-1}$ \\
$n_{M}$ & Manning's friction coefficient & $0 \mathrm{~m}^{-1 / 3} \mathrm{~s}$ \\
$\Delta x$ & Computational cell width & $0.1 \mathrm{~m}$ \\
$\Delta t$ & Simulation time & $200 \mathrm{~s}$ \\
\hline
\end{tabular}

Table 3: Test 3 - Frictionless steady state flow in a non-prismatic trapezoidal channel. Parameters of the test case. 

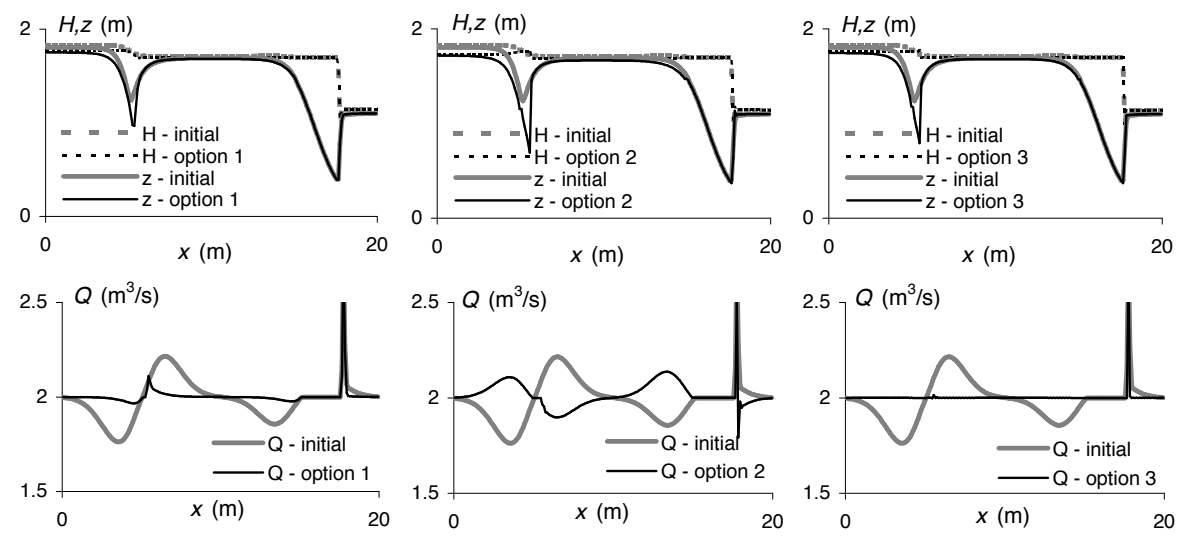

(a) HLL
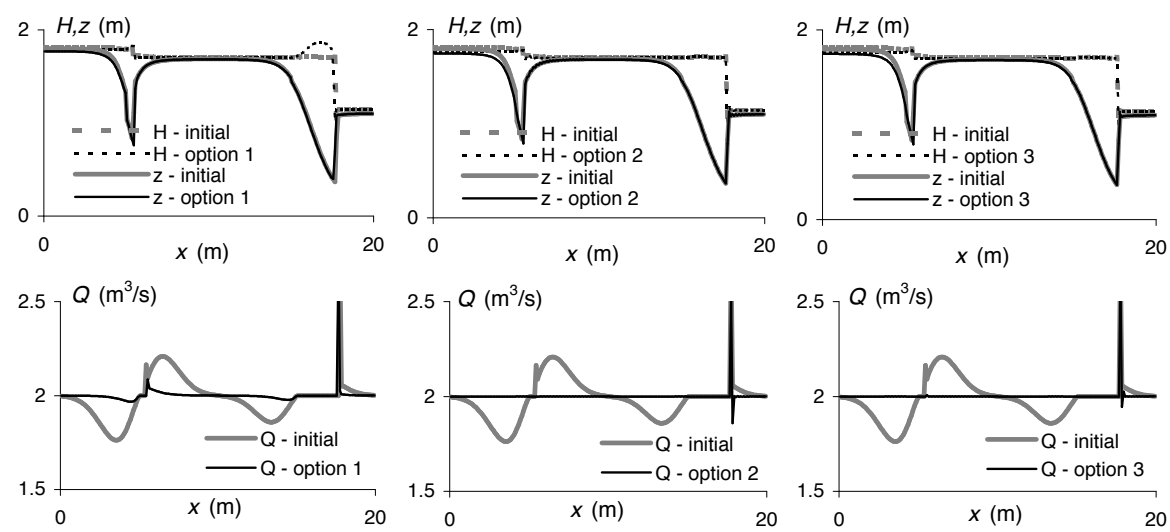

(b) Roe (identical to $Q$-scheme)

Figure 5: Test 2 - Frictionless steady state flow in a non-prismatic, rectangular channel. Up: water elevation $z$ and hydraulic head $H$, down: discharge $Q$ obtained with $\mathbf{V}=\mathbf{U}$ (Initial) and with the three different AVB options, using a) HLL solver, b) Roe's solver. The $Q$-scheme gives similar results to the Roe's solver.
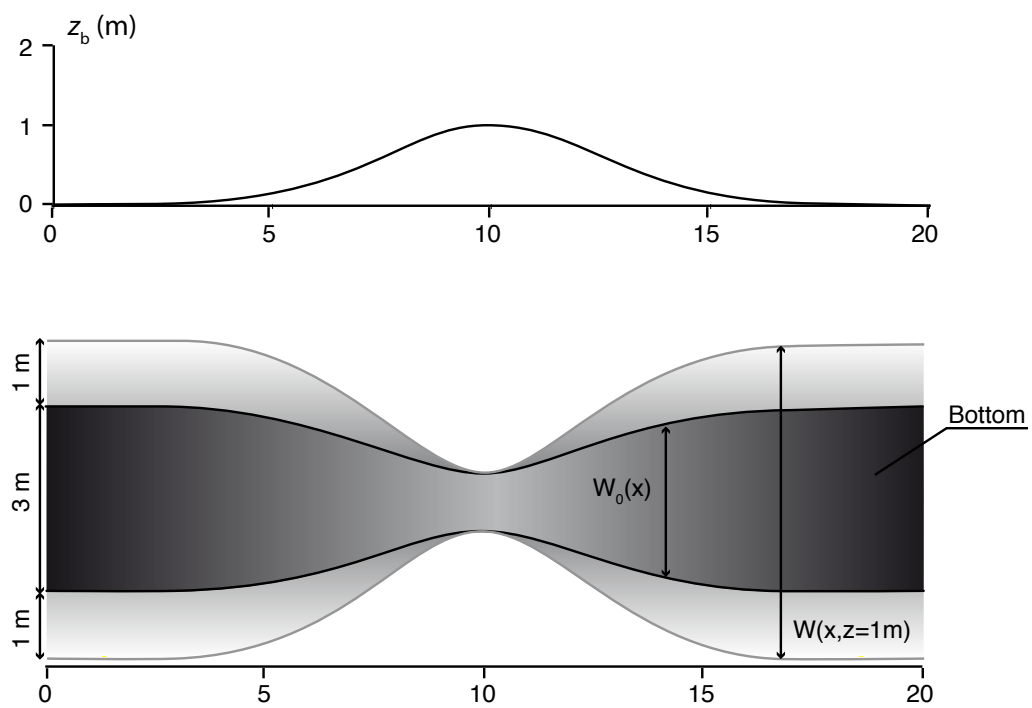

Figure 6: Test 3 - Frictionless steady state flow in a non-prismatic trapezoidal channel. Channel profile: top, bottom elevation; down, $W_{0}(x)$ and $W(x, z=1)$. 
Option 3 gives a uniform value for the discharge everywhere, except across the hydraulic jump, regardless the solver used. Once again, Option 3 is thus deemed more suitable to deal with transcritical flows.

\subsubsection{Test 4: steady state flow in a Venturi flume}

This test case involves the simultaneous presence of all source terms: friction, bottom slope and width variation. It is a real world test case for which experiment validation has been carried out in a channel of $67 \mathrm{~cm}$ wide. The Venturi flume used is $2.5 \mathrm{~m}$ long with narrow section of $10 \mathrm{~cm}$ wide (Figure 8 ). The flume is made of aluminium plates, with a Manning friction coefficient $n_{M}=10^{-2} \mathrm{~m}^{-1 / 3} \mathrm{~s}$ calibrated from experiments in a straight channel made of the same material.

In the experiment, steady state was obtained under a discharge of 40 litres per second. The elevation of the free surface along the walls and axis of the channel was measured every $5 \mathrm{~cm}$. Figure 9 shows numerical results obtained with Roe's solver and the different AVB options. The three AVB options give similar results. The unit-discharge is better estimated upstream than with the initial formulation. Option 2 and 3 give erroneous results with the HLL solver and the $Q$-scheme. Figure 10 shows the longitudinal profiles of the measured and simulated free surface. As can be seen from the figure, the simulation agrees well with the measurement upstream and downstream of the narrowing. In contrast, the free surface elevation is overestimated by the numerical model in the narrow section of the Venturi flume. Besides, the curvature of the simulated free surface profile is wrong. These results invalidate the shallow water assumption of a hydrostatic pressure distribution but this is beyond the scope of the present paper.

\subsection{Transient test cases}

There is no guarantee that an accurate well-balanced approach for steady state flows, gives correct results on transient configurations. The following transient test cases are thus performed.

\subsubsection{Test 5: frictionless dam-break problem in a rectangular channel with flat bottom}

The dam-break problem is an initial-value problem in which the water is initially at rest and the water levels are different on both sides of the dam. The solution of the dam-break problem in rectangular channels is similar to that of the one-dimensional shallow water equations. The properties of the analytical solution are presented in [36]. The dam-break problem is a Riemann problem defined as:

$$
\begin{gathered}
h(x, 0)=\left\{\begin{array}{l}
h_{L} \text { for } x \leq x_{0} \\
h_{R} \text { for } x>x_{0}
\end{array}\right. \\
q(x, 0)=0 \forall x
\end{gathered}
$$

The solution is made of a rarefaction wave and a moving shock separated by a region of constant state. For the dam-break problem without source terms (friction or bottom slope), the profile obeys the following equations in the rarefaction wave

$$
\begin{gathered}
u(x, t)=\frac{2}{3}\left(c_{L}+\frac{x}{t}\right) \\
c(x, t)=\frac{1}{3}\left(2 c_{L}-\frac{x}{t}\right)
\end{gathered}
$$

from which the expression of the flow solution $\mathbf{U}$ is straightforward using $A=c^{2} / g$ and $Q=u A$. In the other parts of the domain, the profile is piecewise constant (see [36] for more details).

The parameters used in this test case are given in Table 4. Profiles of free surface elevation, hydraulic head and discharge, obtained with the initial formulation and the three AVB options are given in Figure 11 for the three solvers. Contrarily to previous test cases, the discharge $Q$ is correctly estimated by each option included initial formulation except for the combinations HLL/Option 2 (Figure 11b) and $Q$-scheme/Option 3 (Figure 11d). For these latter, the free surface elevation and hydraulic head are discontinuous accross the critical point (Note that this problem was pointed out in $[30,18]$ where a specific treatment of the critical point was proposed). 

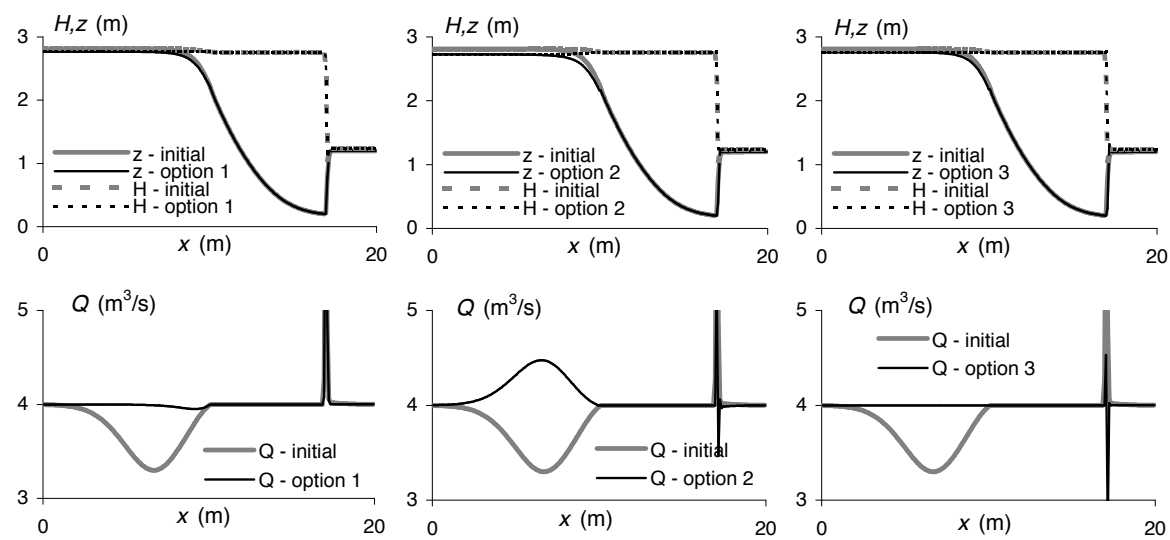

(a) HLL
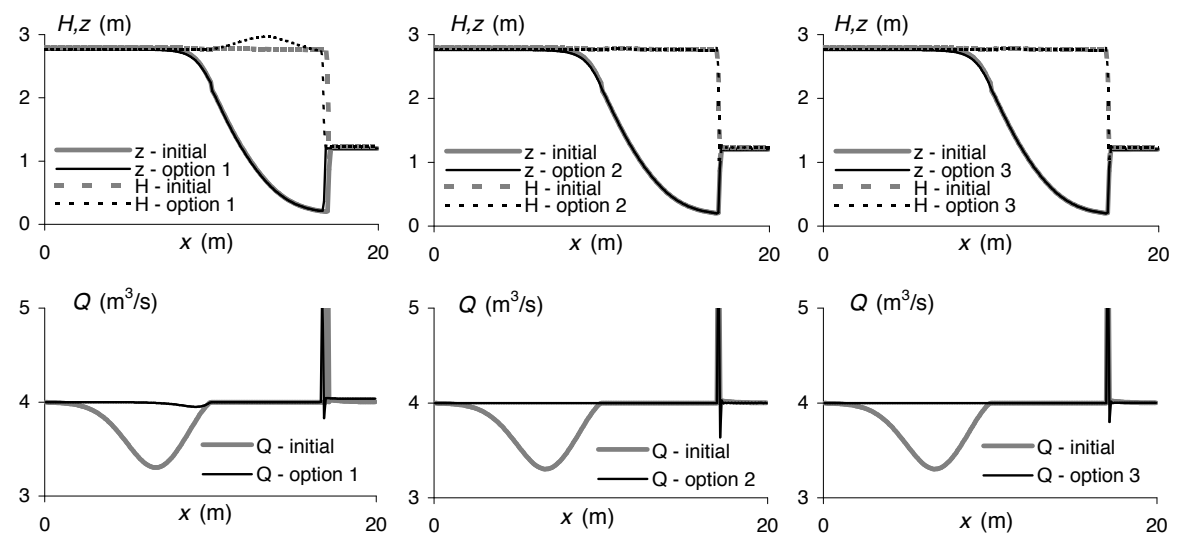

(b) Roe
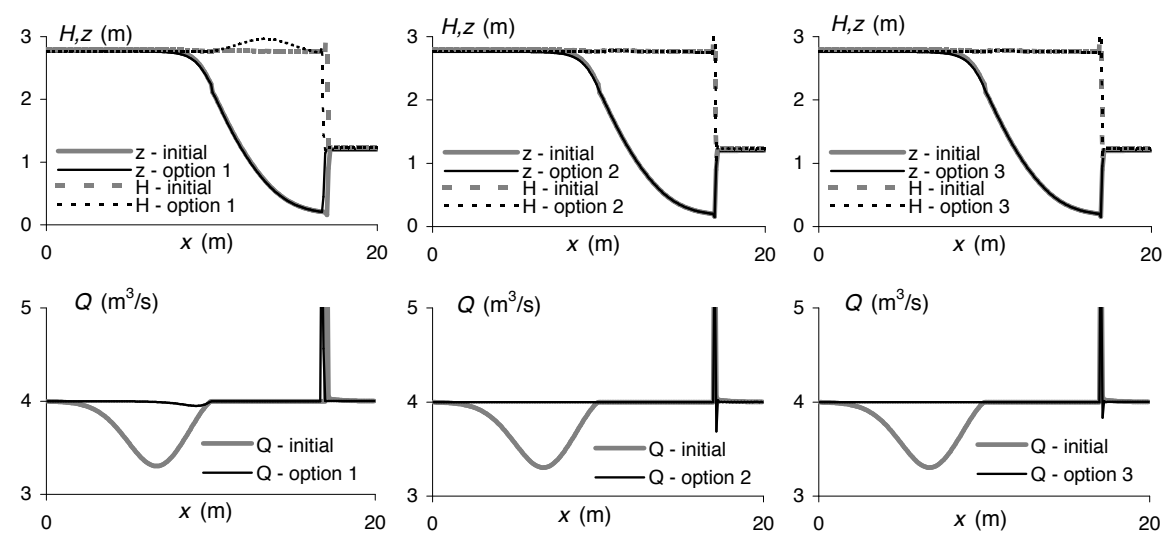

(c) $Q$-scheme

Figure 7: Test 3 - Frictionless steady state flow in a non-prismatic trapezoidal channel. Top: water elevation $z$ and hydraulic head $H$, down: discharge $Q$ obtained with $\mathbf{V}=\mathbf{U}$ (Initial) and with the three different AVB options, using a) HLL solver, b) Roe's solver, c) $Q$-scheme. 

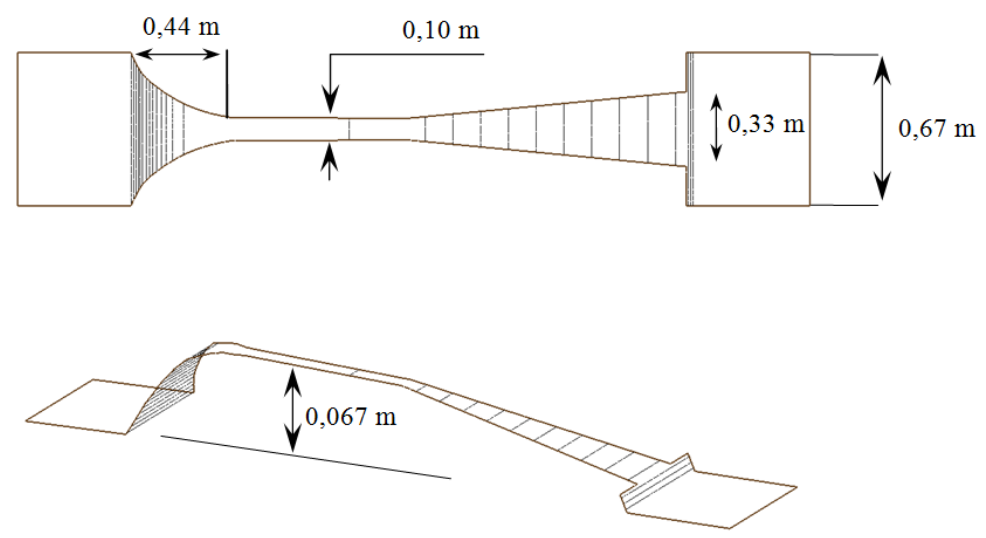

Figure 8: Test 4 - Dimensions of the Venturi flume used in the experiment. Top: plan view. Bottom: bird eye's view with a vertical scale magnified by a factor 5 .
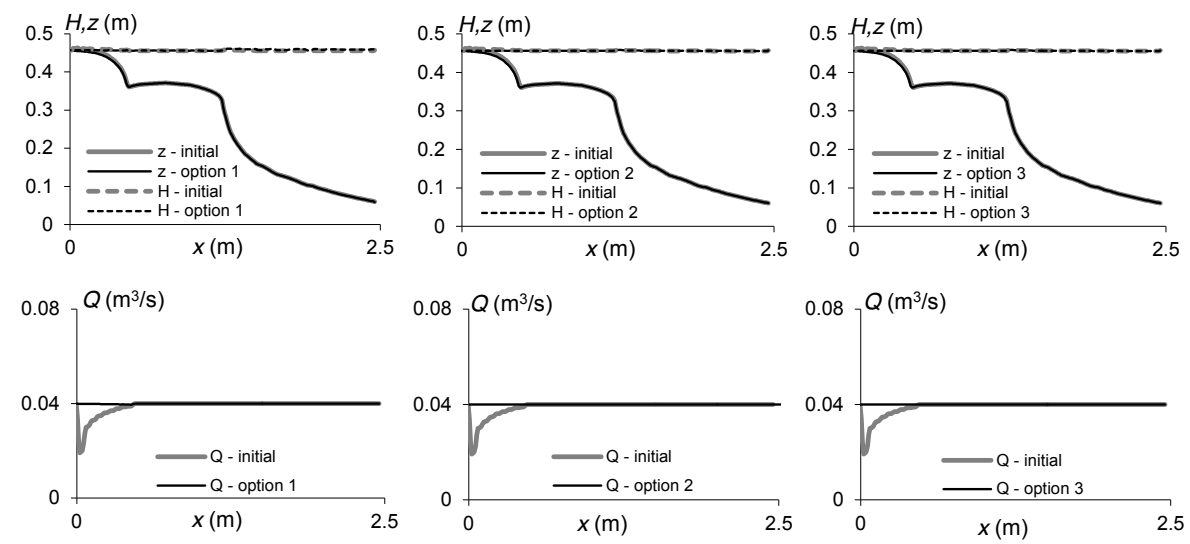

Figure 9: Test 4 - Steady state flow in a Venturi flume. Top: water elevation $z$ and hydraulic head $H$, down: discharge $Q$ obtained with $\mathbf{V}=\mathbf{U}$ (Initial) and with the three different AVB options, using Roe's solver.

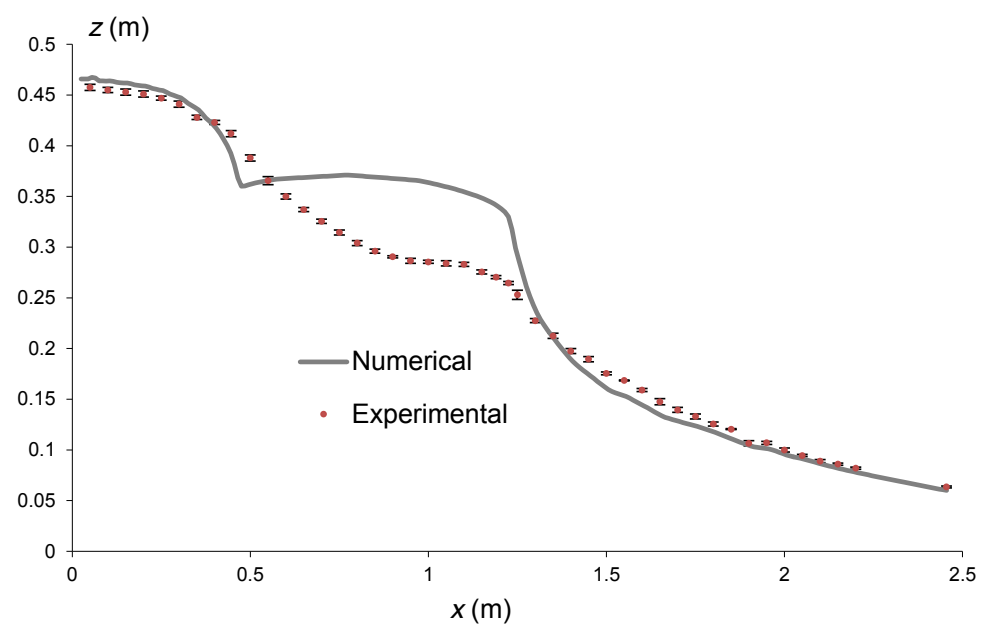

Figure 10: Test 4 - Steady state flow in a Venturi flume. Comparison between numerical results and experimental data. 

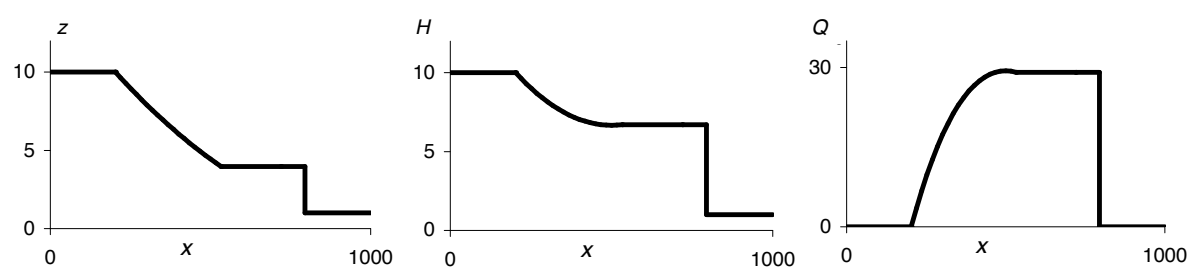

(a) Analytical solution
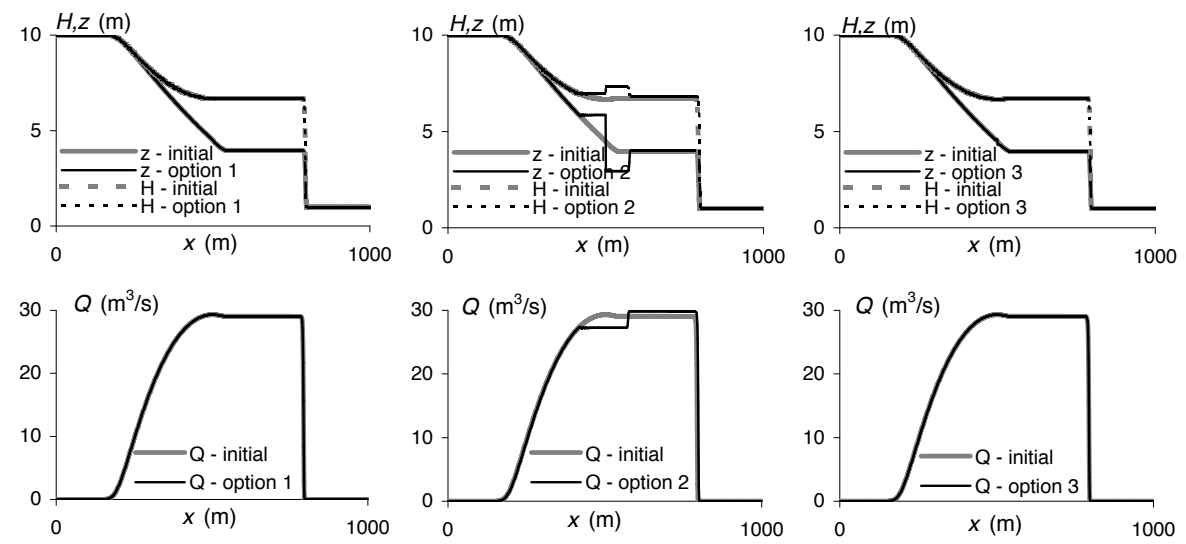

(b) HLL
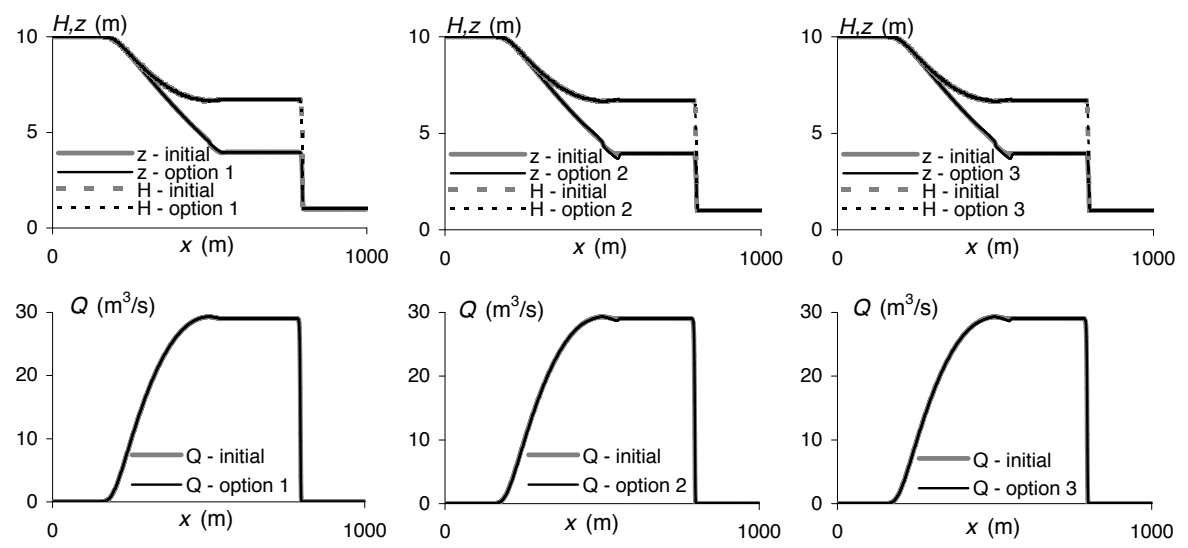

(c) Roe
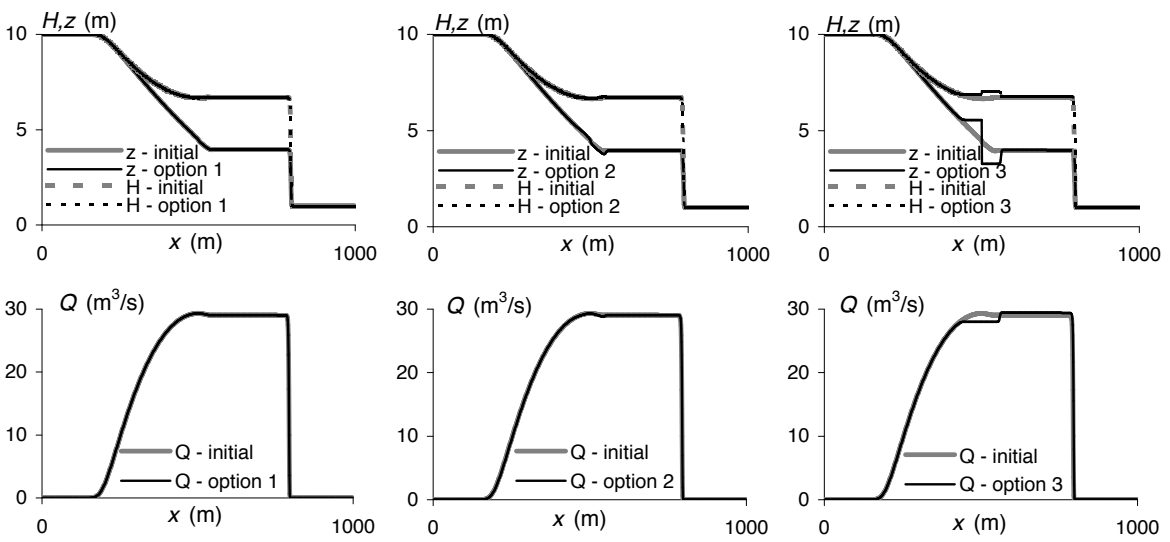

(d) $Q$-scheme

Figure 11: Test 5 - Dam-break problem in a rectangular channel. a) analytical solution; b) HLL solver; c) Roe's solver; d) $Q$-scheme. Top: water surface elevation $z$ and hydraulic head $H$, bottom: discharge $Q$, obtained with $\mathbf{V}=\mathbf{U}$ (Initial) and with the three different AVB options. 


\begin{tabular}{lll}
\hline Symbol & Meaning & Value \\
\hline$g$ & Gravitational acceleration & $9.81 \mathrm{~m} \mathrm{~s}^{-2}$ \\
$L$ & Length of the domain & $1,000 \mathrm{~m}$ \\
$W_{0}$ & Channel width & $1 \mathrm{~m}$ \\
$W_{1}$ & Derivative of the channel width with respect to $z$ & 0 \\
$z_{b}$ & Bottom elevation & $0 \mathrm{~m}$ \\
$h_{L}$ & Initial free surface elevation on the left-hand side of the dam & $10 \mathrm{~m}$ \\
$h_{R}$ & Initial free surface elevation on the right-hand side of the dam & $1 \mathrm{~m}$ \\
$n_{M}$ & Manning's friction coefficient & $0 \mathrm{~m}^{-1 / 3} \mathrm{~s}$ \\
$\Delta x$ & Computational cell width & $1 \mathrm{~m}$ \\
$\Delta t$ & Simulation time & $30 \mathrm{~s}$ \\
\hline
\end{tabular}

Table 4: Test 5 - dam-break problem in a rectangular channel. Parameters of the test case.
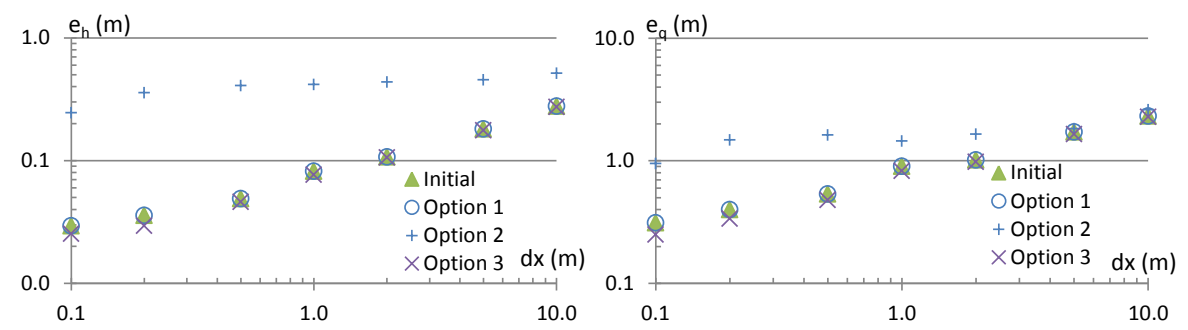

Figure 12: Test 5 - Dam-break problem in a rectangular channel. Convergence analysis using HLL solver and the three AVB options. $L_{2}$-norm between the computed output water depth (left) or unit discharge (right) and analytical solution.

Since the analytical solution is available for the dam-break problem, a convergence analysis is performed on this test case using the three AVB options and HLL solver. Figure 12 shows that options 1 and 3 have almost the same convergence as initial formulation (slightly faster for Option 3), and confirms the non-convergence of Option 2 used with HLL solver.

\subsubsection{Test 6: frictionless dam-break problem in a triangular channel with flat bottom}

This test case is identical to the previous one (dam-break problem in a rectangular channel, without bottom slope or friction) except that the cross-section of the channel has a triangular shape. The parameters of the test case are the same as given in Table 4 for Test 5 except that $W_{0}=0$ and $W_{1}=2$ in the whole domain.

The analytical solution is givent by [25]:

$$
u_{*}+4 c_{*}=u_{L}+4 c_{L}
$$

$$
Q_{*}-Q_{R}=\left(A_{*}-A_{R}\right) c_{s}
$$

$$
\left(\frac{Q^{2}}{A}+\frac{g A^{2}}{2}\right)_{*}-\left(\frac{Q^{2}}{A}+\frac{g A^{2}}{2}\right)_{R}=\left(Q_{*}-Q_{R}\right) c_{s}
$$

where the subscript $*$ denotes the intermediate region of constant state.

Equation (66a) expresses the invariance of the Riemann invariant $(u+4 c)$ across the rarefaction wave. Equations (66b) and (66c) are the jump relationships across the shock moving at the speed $c_{s}$. The unknown shock speed can be eliminated from the system by combining the second and third equations. The system can then be solved iteratively to find the values of $A$ and $Q$ in the intermediate region of constant state using $A=c^{2} / g$ and $Q=u A$. Across the rarefaction wave, $u$ and $c$ verify:

$$
u+4 c=u_{L}+4 c_{L}
$$

$$
u-c=\frac{x}{t}
$$

yielding the following profile for $u$ and $c$ in the rarefaction wave:

$$
u(x, t)=\frac{4}{5}\left(c_{L}+\frac{x}{t}\right)
$$




\begin{tabular}{lll}
\hline Symbol & Meaning & Value \\
\hline$L$ & Length of the domain & $1,000 \mathrm{~m}$ \\
$W_{0}$ & Channel width & $1 \mathrm{~m}$ \\
$W_{1}$ & Derivative of the channel width with respect to $z$ & 0 \\
$z_{b L}$ & Bottom elevation on the left-hand side of the dam & $0 \mathrm{~m}$ \\
$z_{b R}$ & Bottom elevation on the right-hand side of the dam & $5 \mathrm{~m}$ \\
$h_{L}$ & Initial free surface elevation on the left-hand side of the dam & $15 \mathrm{~m}$ \\
$h_{R}$ & Initial free surface elevation on the right-hand side of the dam & $1 \mathrm{~m}$ \\
$n_{M}$ & Manning's friction coefficient & $0 \mathrm{~m}^{-1 / 3} \mathrm{~s}$ \\
$\Delta x$ & Computational cell width & $1 \mathrm{~m}$ \\
$\Delta t$ & Simulation time & $30 \mathrm{~s}$ \\
\hline
\end{tabular}

Table 5: Test 5 - dam-break problem in a rectangular channel. Parameters of the test case.

442

$$
c(x, t)=\frac{1}{5}\left(4 c_{L}-\frac{x}{t}\right)
$$

from which $A$ and $Q$ profiles can be determined.

Results of water elevation $z$, hydraulic head $H$ and discharge $Q$, obtained with the three AVB options and the three solvers are given in Figure 13. In this case again, Roe's solver gives satisfactory results with the 3 options as well as the initial formulation. However, very strong discontinuities at the critical point can be seen with Option 2 and 3 combined with HLL solver and $Q$-scheme, yielding to an underestimation of the maximum discharge. Moreover, the shock is incorrectly located with Option 2/HLL.

\subsubsection{Test 7: frictionless dam-break problem on a bottom step}

The parameters of this test case are given in Table 5 . A bottom step of $5 \mathrm{~m}$ is located at the same abscissa as the initial water depth discontinuity. The analytical solution (that can be found for example in $[1,5])$ as well as results obtained with the three AVB options and Roe's solver are given in Figure 14. HLL solver and $Q$-scheme provide erroneous solutions with Option 2 and 3.

\section{Discussion - Conclusions}

In practical engineering applications, geometrical source terms arising e.g. from bottom slope or the non prismatic character of the channel are to be accounted for in the governing equations. These source terms can in general not be discretized independently of the conservation part. Riemann-solver based techniques compute the fluxes from the average cell values on the left and right hand of the interface. The flux can be seen as a combination of the average cell fluxes, augmented with a diffusion term involving the gradient in the conserved variable. Artificial oscillations may appear in the computed profiles if the gradients (and hence the diffusive part of the flux) is not estimated properly. The Auxiliary Variable-Based balancing, consists of using an "auxiliary" variable instead of the conserved one in the flux function, defined so as to allow the steady-state condition (of which static equilibrium is only a particular case) to be preserved. It is applied to the one-dimensional open channel equations in the present paper.

Three different options of AVB have been tested in this paper in addition to the classical flux formulation that uses the gradient of the conserved variables: 1) free surface elevation; 2) specific force and 3) hydraulic head. The application of the method to three classical approximate Riemann solvers (HLL, Roe and $Q$-scheme) is also presented.

Various steady-state test cases including singular head losses, friction, bottom and width variation, non-prismatic configurations, have been implemented to assess the ability of the AVB approach to deal with transcritical flows, the critical points being well-known to introduce instabilities. In the steady-state test cases, the three options generally gives a better estimate of the uniform discharge than the initial formulation. Option 3, based on the hydraulic head, is the one that gives a uniform discharge equal to the prescribed one with the best accuracy, for each steady-state test case and in the whole domain, except across hydraulic jumps where a small spike remains. It is important to check the validity of these approaches for unsteady states configurations. Indeed, some examples in the literature that give correct results in steady state configurations (such as [28]) have revealed incorrect on 

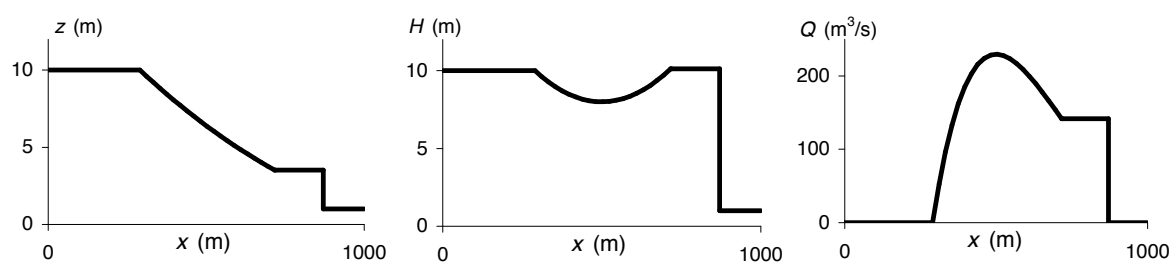

(a) Analytical solution
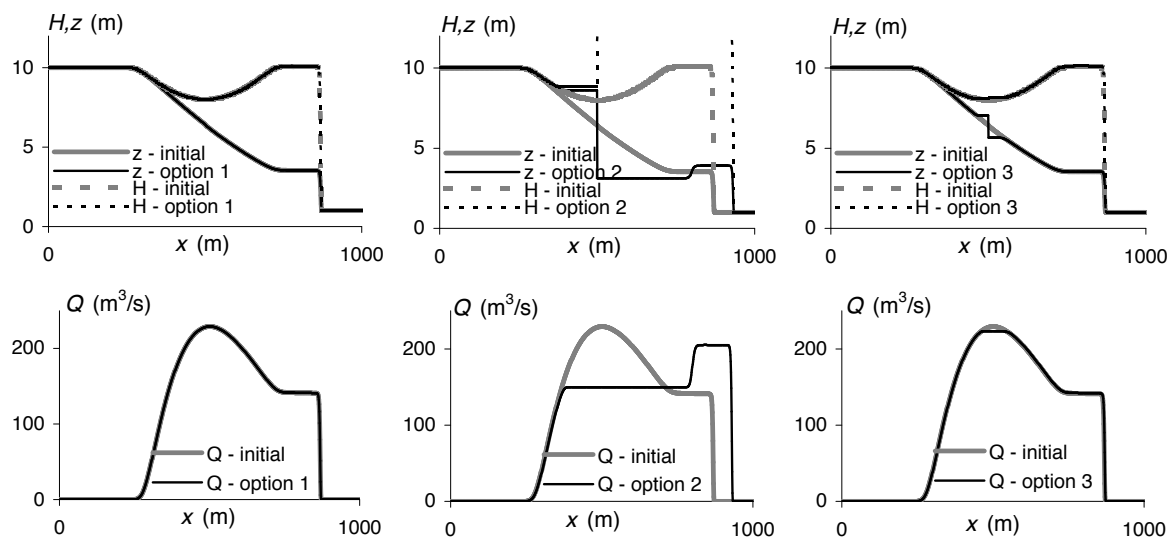

(b) HLL
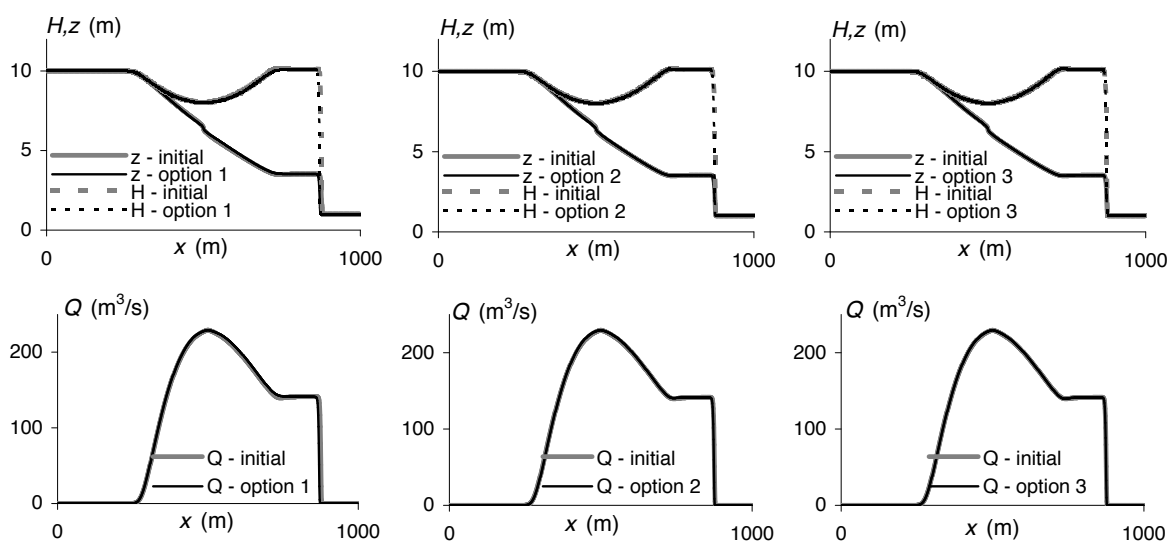

(c) Roe
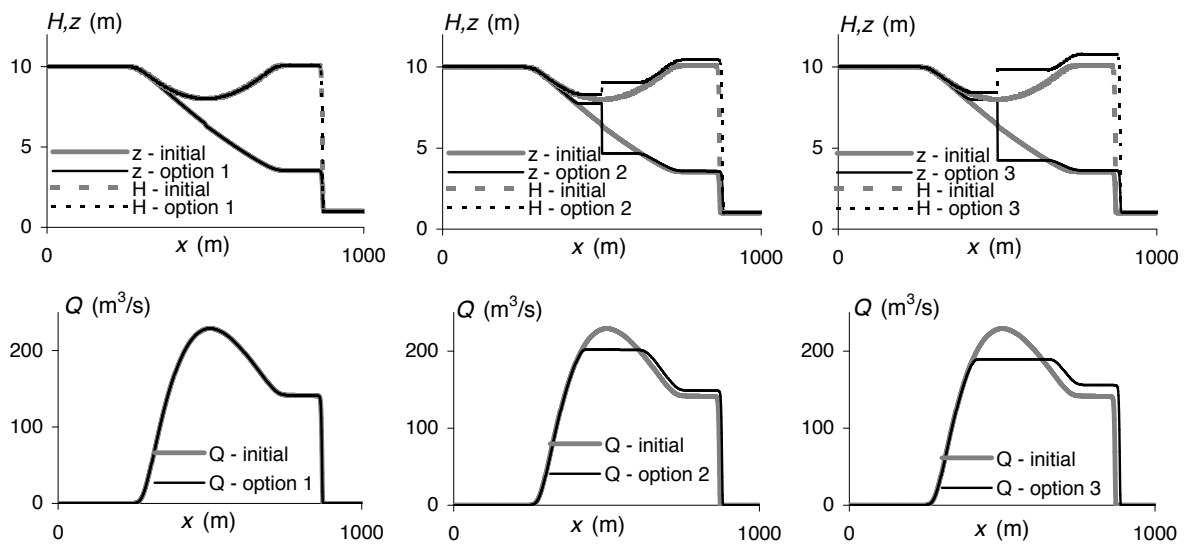

(d) $Q$-scheme

Figure 13: Test 6 - Dam-break problem in a triangular channel. a) Analytical solution, b) HLL solver, c) Roe's solver and d) $Q$-scheme. For each sub-figure, top: hydraulic head $H$ and water elevation $z$, bottom: discharge $Q$, obtained with $\mathbf{V}=\mathbf{U}$ (Initial) and with the three different AVB options. 

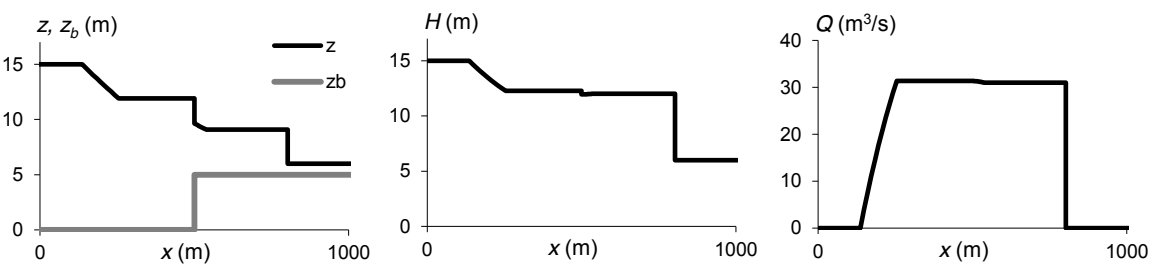

(a) Analytical solution
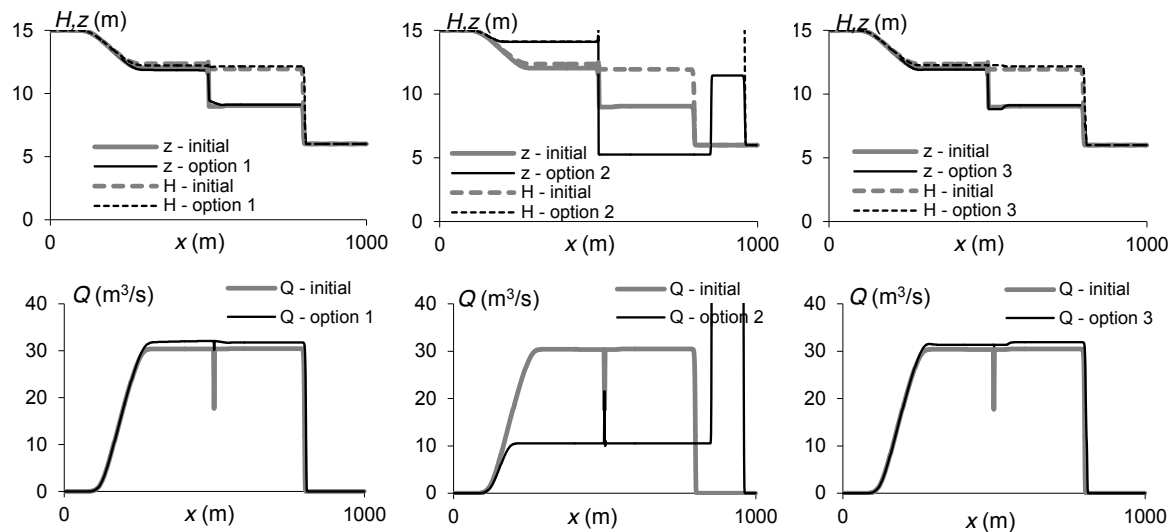

(b) Roe

Figure 14: Test 07 - Dam-break problem on a bottom step. a) Analytical solution; b) top: water surface elevation $z$ and hydraulic head $H$, bottom: discharge $Q$, obtained using Roe's solver with $\mathbf{V}=\mathbf{U}$ (Initial) and with the three different AVB options.

transient test cases, such as shown in the three unsteady configurations presented (dambreak in rectangular or triangular channels and over a bottom step) where Option 2 gives bad results when used with HLL or $Q$-scheme.

Finally, when used with Roe's solver, Option 3 is the only one that produces correct results for all the test cases. An interesting feature of this option is that it allows head loss functions (stemming from e.g. bridges or other singularities) to be accounted for directly within the discretized equations. In contrast with what is classically done in commercially available river packages, the AVB method eliminates the need for internal boundaries across hydraulic singularities.

In the present paper, the geometric source term is accounted for by integrating the bottom slope over the surface of the (non-horizontal) computational cell. This procedure is rather easy to carry out when the cell is full. But in the case of wetting/drying, the water does not occupy the full length of the cell. Computing the integral of the term $g h S_{0}$ over only part of the cell becomes a very complex and time-consuming task. The approach to source term estimation proposed in the present paper is thus not the best possible option to the discretization of real-world geometries and practical river problems. An alternative option is currently under study. It consists in considering each cell as prismatic and lumping the geometric source term at the cell interfaces. This approach, however, requires that a proper splitting of the lumped source terms between the adjacent cells to the interfaces be devised. This point is currently under study.

Finally, this paper deals with finite volume Godunov-type discretizations, but if higherorder schemes are to be designed, the AVB method may be applied by reconstructing the auxiliary variable.

\section{References}

[1] F. Alcrudo and F. Benkhaldoun. Exact solutions to the Riemann problem of the shallow water equations with a bottom step. Computers $\mathcal{E}$ Fluids, 30:643-671, 2001.

[2] E. Audusse and M.-O. Bristeau. A well-balanced positivity preserving "second-order" 
scheme for shallow water flows on unstructured meshes. Journal of Computational Physics, 206:311-333, 2005.

[3] F. Benkhaldoun, I. Elmahi, and M. Seaid. A new finite volume method for flux-gradient and source-term balancing in shallow water equations. Computer Methods in Applied Mechanics and Engineering, 199:3324-3335, 2010.

[4] A. Bermudez and M.E. Vazquez. Upwind methods for hyperbolic conservation laws with source terms. Computers \& Fluids, 23:1049-1071, 1994.

[5] R. Bernetti, V.A. Titarev, and E.F. Toro. Exact solution of the Riemann problem for the shallow water equations with discontinuous bottom geometry. Journal of Computational Physics, 227:3212-3243, 2008.

[6] J. Burguete and P. Garcia-Navarro. Improving simple explicit methods for unsteady open channel and river flow. International Journal for Numerical Methods in Fluids, 45:125-156, 2004.

[7] V. Caleffi, A. Valiani, and A. Bernini. Fourth-order balanced source term treatment in central weno schemes for shallow water equations. Journal of Computational Physics, 218(1):228 - 245, 2006.

[8] A. Canestrelli, M. Dumbser, A. Siviglia, and E.F. Toro. Well-balanced high-order centered schemes on unstructured meshes for shallow water equations with fixed and mobile bed. Advances in Water Resources, 33(3):291-303, MAR 2010.

[9] H. Capart, T.I. Eldho, S.Y. Huang, D.L. Young, and Zech Y. Treatment of natural geometry in finite volume river flow computations. Journal of Hydraulic Engineering ASCE, 129:385-393, 2003.

[10] M.J. Castro, J.M. Gallardo, and C. ParÈs. High-order finite volume schemes based on reconstruction of states for solving hyperbolic systems with nonconservative products. applications to shallow-water systems. Mathematics of Computation, 75:1103-1134, 2006.

[11] T. Chacon Rebollo, A. Dominguez Delgado, and E.D. Fernandez Nieto. A family of stable numerical solvers for the shallow water equations with source terms. Computer Methods in Applied Mechanics and Engineering, 192:203-225, 2003.

[12] T. Chacon Rebollo, A. Dominguez Delgado, and E. Fernandez Nieto. Asymptotically balanced schemes for non-homogeneous hyperbolic systems - application to the shallow water equations. Comptes Rendus de l'Académie des Sciences Serie I, 338:85-90, 2004.

[13] Nelida Crnjaric-Zic and Bojan Crnkovic. High order accurate semi-implicit WENO schemes for hyperbolic balance laws. Applied mathematics and computation, 217(21): 8611-8629, JUL 12011.

[14] J.A. Cunge, F.M. Jr Holly, and A. Verwey. Practical Aspects of River Computational Hydraulics. Pitman, 1980.

[15] S.F. Davis. Simplified second-order Godunov-type methods. SIAM Journal of Scientific and Statistical Computing, 9:445-473, 1988.

[16] M. Dumbser and E.F. Toro. A simple extension of the osher riemann solver to nonconservative hyperbolic systems. Journal of Scientific Computing, 48:70-88, 2011.

[17] B. Einfeldt. On Godunov-type methods for gas dynamics. SIAM Journal of Numerical Analysis, 25:294-318, 1988.

[18] P. Finaud-Guyot, C. Delenne, J. Lhomme, V. Guinot, and C. Llovel. An approximatestate Riemann solver for the two-dimensional shallow water equations with porosity. International Journal for Numerical Methods in Fluids, 62:1299-1331, 2010.

[19] T. Gallouet, J.M. Herard, and N. Seguin. On the use of symmetrizing variables for vacuums. CALCOLO, 40:163-194, 2003. 
[20] T. Gallouet, J.M. HĖrard, and N. Seguin. Some approximate godunov schemes to compute the shallow-water equations with topography. Computers \& Fluids, 32:479$513,2003$.

[21] P. Garcia-Navarro and M.E. Vazquez-Cendon. On numerical treatment of the source terms in the shallow water equations. Computers \& Fluids, 29:951-979, 2000.

[22] P. Glaister. Approximate Riemann solutions of the shallow water equations. Journal of Hydraulic Research, 26:293-306, 1988.

[23] S.K. Godunov. A difference method for calculation of discontinuous solutions of hydrodynamics. Matematicheski Sbornik, 47:271-306, 1959.

[24] J.M. Greenberg and A. Leroux. A well-balanced scheme for the numerical processing of source terms in hyperbolic equations. SIAM Journal of Numerical Analysis, 33:1-16, 1996.

[25] V. Guinot. Wave Propagation in Fluids. Models and numerical techniques. 2nd edition. Wiley-ISTE, 2010.

[26] A. Harten, P.D. Lax, and B. Van Leer. On upstream differencing and Godunov-type schemes for hyperbolic conservation laws. Journal of Computational Physics, 50:235$269,1983$.

[27] G. Kesserwani and Q. Liang. Well-balanced RKDG2 solutions to the shallow water equations over irregular domains with wetting and drying. Computers and Fluids, 39: $2040-2050,2010$.

[28] S.H. Lee and N.G. Wright. Simple and efficient solution of the shallow water equations with source terms. International Journal for Numerical Methods in Fluids, 63:313-340, 2010 .

[29] R.J. LeVeque. Balancing source terms and flux gradients in high-resolution Godunov methods: the quasi-steady wave propagation algorithm. Journal of Computational Physics, 146:346-365, 1998.

[30] J. Lhomme and V. Guinot. A general approximate-state solver for hyperbolic systems of conservation laws with source terms. International Journal for Numerical Methods in Fluids, 53:1509-1540, 2007.

[31] Q. Liang and F. Marche. Numerical resolution of well-balanced shallow water equations with complex source terms. Advances in Water Resources, 32:873-884, 2009.

[32] J. Murillo, P. García-Navarro, J. Burguete, and P. Brufau. The influence of source terms on stability, accuracy and conservation in two-dimensional shallow flow simulation using triangular finite volumes. International Journal for Numerical Methods in Fluids, 54 (5):543-590, 2007.

[33] M. Nujic. Efficient implementation of non-oscillatory schemes for the computation of free-surface flows. Journal of Hydraulic Research, 33:101-111, 1995.

[34] P.L. Roe. Approximate Riemann solvers, parameter vectors and difference schemes. Journal of Computational Physics, 43:357-372, 1981.

[35] L. Song, J. Zhou, J. Guo, Q. Zou, and Y. Liu. A robust well-balanced finite volume model for shallow water flows with wetting and drying over irregular terrain. Advances in Water Resources, 34(7):915-932, JUL 2011.

[36] J.J. Stoker. Water Waves. Interscience, 1957.

[37] A Valiani and L Begnudelli. Divergence form for bed slope source term in shallow water equations. Journal of Hydraulic Engineering - ASCE, 132(7):652-665, JUL 2006.

[38] M.E. Vazquez-Cendon. Improved treatment of source terms in upwind schemes for the shallow water equations in channels with irregular geometry. Journal of Computational Physics, 148:497-526, 1999. 
${ }_{607}^{6}$ [39] Y. Xing and C.-W. Shu. High order well-balanced finite volume weno schemes and

608

609

610

611

612 discontinuous galerkin methods for a class of hyperbolic systems with source terms. Journal of Computational Physics, 214(2):567 - 598, 2006.

[40] J.G. Zhou, D.M. Causon, C.G. Mingham, and D.M. Ingram. The surface gradient method for the treatment of source terms in the shallow water equations. Journal of Computational Physics, 168:1-25, 2001. 